\title{
Diatomic molecules and fermionic particles with improved Hellmann-generalized Morse potential through the solutions of the deformed Klein-Gordon, Dirac and Schrödinger equations in extended relativistic quantum mechanics and extended nonrelativistic quantum mechanics symmetries
}

\author{
A. Maireche \\ Department of Physics, M'sila University, Laboratory of Physics and Material Chemistry, \\ M'sila University, BP 239, Algeria. \\ *e-mail: abdelmadjid.maireche@univ-msila.dz
}

Received 6 May 2021; accepted 7 June 2021

\begin{abstract}
In this paper, we investigate the new approximate bound state solution of deformed Klein-Gordon, Dirac and Schrödinger equations in the symmetries of extended relativistic quantum mechanics ERQM and extended nonrelativistic quantum mechanics ENRQM have been obtained with a newly proposed potential called improved Hellmann-generalized Morse potential (IHGMP, for short). To the best of our knowledge, this problem is examined in literature in the usual RQM and NRQM with Hellmann-generalized Morse potential. The potential is a superposition of Hellmann potential, generalized Morse or Deng-Fan potential, and some other exponential terms. By employing the improved approximation to deal with the centrifugal term, Bopp's shift and standard perturbation theory method. The new approximate analytical energy shift and the corrections of bound state energy eigenvalues in ERQM and ENRQM are obtained for some selected diatomic molecules such as $(\mathrm{HCl}, \mathrm{LiH}, \mathrm{H} 2, \mathrm{ScH}, \mathrm{TiH}, \mathrm{VH}, \mathrm{CrH}, \mathrm{CuLi}, \mathrm{TiC}, \mathrm{NiC}, \mathrm{ScN}$ and $\mathrm{ScF})$. The new values that we get are sensitive to the quantum numbers $(j, l, s, m)$, the potential depths of the improved Hellmann-generalized Morse potential $(a, b)$, the range of the potential $\alpha$, the dissociation energy $D_{e}$, the equilibrium bond length $r_{e}$, and noncommutativity parameters $(\Theta, \sigma, \chi)$. We have highlighted three physical phenomena that automatically generate a result of the topological properties of noncommutativity, the first physical phenomena are the perturbative spin-orbit coupling, the second the magnetic induction while the third corresponds to the rotational proper phenomena. In both relativistic and nonrelativistic problems, we show that the corrections on the spectrum energy are smaller than the main energy in the ordinary cases of quantum field theory and quantum mechanics. In the new symmetries of NCQM, it is not possible to get the exact analytical solutions for $l=0$ and $l \neq 0$, the approximate solutions are available. Four special cases, i.e., 1 wave are investigated in the context of deformed Klein-Gordon and Schrödinger theories. The relativistic energy equations and the new nonrelativistic energy for some potentials such as improved Hellmann potential and improved generalized Morse potential have also been obtained by varying some potential parameters. We have clearly shown that the Schrödinger and Klein Gordon equations in the new symmetries can physically describe each of the two Dirac equations and the Duffin-Kemmer equation under the effect of IHGMP.
\end{abstract}

Keywords: Klein-Gordon equation; Schrödinger equation; Morse potential; Hellmann potential; the diatomic molecules; noncommutative geometry; star products; Bopp's shift method.

DOI: https://doi.org/10.31349/RevMexFis.68.020801

\section{Introduction}

Two scientific revolutions took place at the beginning of the last century; the first was embodied by the general and special theories of relativity and the second was the development of quantum mechanics. The Schrödinger equation was the first used to probe matter at the smallest scales [1], after which came the Klein-Gordon, Duffin-Kemmer, and Dirac equations. For the case of neutral or charged particles with spin zero, one can deal with the Klein-Gordon equation [2,3]. In contrast, for the case of a particle with spin-1, the DuffinKemmer equation [4] is necessary for mesons. As for the case in the case of spin-1/2 as electrons and their antagonists (positron), the Dirac equation [5] is the tool used to access the physical and chemical information of the system. All fundamental equations that we have referred to are normally solved using different potentials, depending on the nature of the problem being studied. Exponential potentials have been, and still are, the tool through which researchers have sought to study molecules. We will devote our current study to two types of potentials of great importance in this field, the Hellmann and generalized Morse potential. Many researchers have previously studied them in different energy levels, either separately for each of them or in combination, but it was in the framework of usual nonrelativistic quantum mechanics (NRQM) and relativistic quantum mechanics (RQM). Our study will be the focus of a case for combining them in the framework of a large quantum symmetry that is known by nonrelativistic noncommutative quantum mechanics (or extended nonrelativistic quantum mechanics, ENRQM) and relativistic noncommutative quantum mechanics (ERQM) symmetries (or extended relativistic quantum mechanics) using deformed Schrödinger, Klein-Gordon, and Dirac equations. 
It is well known that the Hellmann potential [6-8], is a superposition of the attractive Coulomb potential and a Yukawa potential [9]. Ikhdair et al. [10] have studied the bound state energies of the Hellmann potential by using the SUSY perturbation formalism. In 2007, Koncak et al. [11] studied the radial Schrödinger equation for the Hellmann potential within the framework of the asymptotic iteration method and obtained the bound state energy eigenvalues. This potential has many important applications; we mention one of the solidstate physics [12-14], alkali hydride molecules [15], innershell ionization problem [16], nuclear physics [17], among other applications. Furthermore, it was studied in both relativistic and non-relativistic quantum mechanics (see, e.g., [17-20]).

Deng-Fan potential originally appeared many decades ago. This potential was proposed by Deng and Fan [21] as a molecular potential, which improved the Morse potential [22], is known as the generalized Morse potential. Dong has used this potential as a suitable alternative potential to the Morse potential in the study of diatomic molecules to describe the vibrational spectrum and electromagnetic transitions $[23,24]$. Moreover, it can be used to study the diatomic molecular and obtain their energy spectra [25]. In 2008, Dong and $\mathrm{Gu}$ [26] obtained a bound state solution of the Schrödinger equation with the Deng-Fan molecular potential. Dong [27] trained the relativistic of spinless particles subject to a rotating Deng-Fan Oscillator. Oluwadare et al. [24] obtained the exact $l$-wave solutions of the Klein-Gordon and Dirac equations with equally mixed scalar and vector DengFan molecular potentials, the normalized wave function, and the corresponding energy equations. Hassanabadi et al. analyzed the relativistic spinless particles under Deng-Fan potential [28]. By using the asymptotic iteration method, Ortakaya et al. [29] obtained the approximate analytical solutions of the Dirac equation with the Deng-Fan potential including a Coulomb tensor interaction in the presence of spin symmetry and pseudo-spin symmetry. In 2009, Zhang et al. [30] obtained the approximate analytical solutions of the Dirac equation with the generalized Morse potential model in the presence of spin symmetry and pseudo-spin symmetry by using the supersymmetric shape invariance formalism. Also, Daif [31] obtained $l$-state solutions of the Feynman propagator with the Deng-Fan molecular potential. Moreover, Maghsoodi et al. obtained. By employing the Pekeris-type approximation, Oyewumi et al. [32] obtained bound state solutions of the Deng-Fan molecular potential using the NikiforovUvarov method for diatomic molecules $(\mathrm{HCl}, \mathrm{LiH}, \mathrm{H} 2, \mathrm{ScH}$, $\mathrm{TiH}, \mathrm{VH}, \mathrm{CrH}, \mathrm{CuLi}, \mathrm{TiC}, \mathrm{NiC}, \mathrm{ScN}$ and $\mathrm{ScF}$ ). Very recently, Ekwevugbe [33] obtained a nonrelativistic energy spectrum of the Deng-Fan Oscillator via the WKB approximation. Currently, the idea of combining more than two potentials has attracted interest. This combination expands the application scope to include new fields. And as a successful model for this combination, Okoia et al. and Ebomwonyi et al. studied the Hellmanngeneralized and Morse potentials in the case of the relativistic Klein-Gordon equation, Dirac equation, and nonrelativistic Schrödinger equation; this combination can be applied in different branches of physics, including molecular and atomic physics [34,35].

In recent work, we combine Hellmann-generalized Morse potentials and explore the corresponding deformed KleinGordon, Dirac, and Schrödinger equations in the symmetries of ERQM and ENRQM. The idea of non-commutative quantum mechanics is old and dates to the early years of ordinary quantum mechanics, originally from Snyder [36] and later developed. The non-commutative quantum theory contributed positively to overcoming many problems that ordinary quantum mechanics could not solve. Quantum gravity, string theory, and the divergence problem of the standard model new data made this new quantum theory a refuge for physicists to find solutions and discoveries hoped for on the other hand [38-48]. In recent years, a lot attention has been drawn to this development [49-59].

Concerning the combination of Hellmann and generalized Morse potentials, the subject of the current study, I have previously dealt with the non-specific study of each of them separately, but I have not dealt with them in a combined way, and neither has any other researcher done so far. We have treated the generalized Hellmann potential in the symmetries of NERQM [60]. Moreover, we have applied the Hellmann potential on the Mirror Nuclei ${ }^{17} \mathrm{O}$ and ${ }^{17} \mathrm{~F}$ in the symmetries of NERQM [61]. Moreover, we have studied the deformed Schrödinger equation with the generalized Hellmann-Kratzer potential model in the symmetries of ENRQM [62]. Very recently, we have investigated the bound-state solutions of the deformed Klein-Gordon and Schrödinger equations for arbitrary l-states with the modified Morse potential in the symmetries of noncommutative quantum mechanics [54]. From what we have seen so far that most of the studies concerning improved Hellmann-generalized Morse potential were within the framework of ordinary quantum mechanics. The above works motivated us to investigate the approximate solutions of the 3-dimensional deformed Klein-Gordon equation, Dirac and Schrödinger equations for improved Hellmann-generalized Morse potential offered in Refs. [34, 35] in RQM and NRQM. The potential focus of study and interest can be applied for some selected diatomic molecules such as $(\mathrm{HCl}, \mathrm{LiH}, \mathrm{H} 2, \mathrm{ScH}, \mathrm{TiH}, \mathrm{VH}, \mathrm{CrH}, \mathrm{CuLi}$, TiC, NiC, ScN and $\mathrm{ScF}$ ) in ERQM and ENRQM symmetries. The research reported in the present article was motivated by the fact that the study of the MHGPs in the ERQM and ENRQM symmetries has not been reported in the available literature. Here, our focus was on the MHGPs, which has the following form in the new symmetry:

$$
\begin{aligned}
(V, S)_{\mathrm{hmp}}(r) & =\left(D_{e}, S_{e}\right)\left[1+\frac{-a+b e^{-\alpha r}}{r\left(D_{e}, S_{e}\right)}\right. \\
& \left.-2\left(\frac{e^{\alpha r_{e}}-1}{e^{\alpha r}-1}\right)+\left(\frac{e^{\alpha r_{e}}-1}{e^{\alpha r}-1}\right)^{2}\right],
\end{aligned}
$$




$$
\begin{aligned}
(V, S)_{\mathrm{hmp}}\left(r_{n c}\right) & =(V, S)_{\mathrm{hmp}}(r) \\
& -\frac{\partial(V, S)_{\mathrm{hmp}}(r)}{\partial r} \frac{\mathbf{L} \Theta}{2 r}+O\left(\Theta^{2}\right),
\end{aligned}
$$

where $\left(D_{e}, S_{e}\right)$ are the dissociations energies, $r_{e}$ is the equilibrium bond length, ( $a$ and $b$ ) are the potential strengths, $\alpha$ is the screening parameter, $r_{n c}$ and $r$ is the distance between the two particles in EQM and QM symmetries, respectively. The coupling equals $\mathbf{L} \Theta \equiv \vec{L} \vec{\Theta}$ equals $L_{x}, \Theta_{12}+L_{y} \Theta_{23}+L_{z}$ $\Theta_{13}$ with $L_{x}, L_{y}$ and $L_{z}$ are present the usual components of the angular momentum operator $\mathbf{L}$ while the new noncommutativity parameter $\Theta_{i j}$ equals $\theta_{i j} / 2$. The new algebraic structure of noncommutative covariant canonical commutations relations NCNCCRs in the three representations of Schrödinger, Heisenberg, and interactions pictures, as follows (It should be noted that, in our calculation, we have used the natural units $\hbar=c=1$ ) [63-70]:

$$
\begin{gathered}
\left\{\begin{array}{c}
{\left[x_{\mu}^{(S, H, I)}, p_{\nu}^{(S, H, I)}\right]=i \hbar \delta_{\mu \nu}} \\
\Longrightarrow\left[x_{n c \mu}^{(S, H, I)^{*}, p_{n c \nu}(S, H, I)}\right]=i \hbar \delta_{\mu \nu} \\
{\left[x_{\mu}^{(S, H, I)}, x_{\nu}^{(S, H, I)}\right]=0} \\
\Longrightarrow\left[x_{n c \mu}^{(S, H, I)_{*}}, x_{n c \nu}^{(S, H, I)}\right]=i \theta_{\mu \nu}
\end{array}\right.
\end{gathered}
$$

While the uncertainty relations will be changed into the following formula in the new symmetries as follows:

$$
\begin{aligned}
\left|\Delta x_{\mu}^{(S, H, I)} \Delta p_{\nu}^{(S, H, I)}\right| \geqslant \frac{\hbar \delta_{\mu \nu}}{2} \\
\Longrightarrow\left\{\begin{array}{l}
\Delta x_{\mu}^{(S, H, I)} \Delta p_{\nu}^{(S, H, I)} \geqslant \frac{\hbar_{e f f} \delta_{\mu \nu}}{2} \\
\left|\Delta x_{\mu}^{(S, H, I)} \Delta p_{\nu}^{(S, H, I) \mid \geqslant \frac{\left|\hbar \theta_{\mu \nu}\right|}{2}}\right|
\end{array} .\right.
\end{aligned}
$$

With $x_{n c \mu}^{(S, H, I)}=\left(x_{n c \mu}^{S}, x_{n c \mu}^{H}, x_{n c \mu}^{I}\right)$ are the generalized coordinates in NCQM symmetries and $p_{n c \mu}^{(S, H, I)}=$ $\left(p_{n c \mu}^{S}, p_{n c \mu}^{H}, p_{n c \mu}^{I}\right)$ are the corresponding generalizing coordinates in the usual QM symmetries. It is important to note that Eq. (2.2) is a covariant equation (the same behavior of $x_{\mu}^{(S, H, I)}$ ) under Lorentz transformation, which includes boosts and/or rotations of the observer's inertial frame. We are generalizing the NCNCCRs to include Heisenberg and interaction pictures. Here $\hbar_{\text {eff }} \cong \hbar$ is the effective Planck constant, $\theta_{\mu \nu}=\epsilon_{\mu \nu} \theta$ ( $\theta$ is the non-commutative parameter) which is an infinitesimal parameter if compared to the energy values and elements of antisymmetric $3 \times 3$ real matrices and $\delta_{\mu \nu}$ is the identity matrix. The symbol $*$ denotes the Weyl Moyal star product, which is generalized between two ordinary functions $f(x) h(x)$ to the new deformed form $\hat{f}\left(x_{n c}\right) \hat{h}\left(x_{n c}\right)$ which expressed with the Weyl Moyal star product $f(x) * h(x)$ in the symmetries of NCQM as follows $\theta_{\mu \nu}=\epsilon_{\mu \nu} \theta$ ( $\theta$ is the non-commutative parameter) which is an infinitesimal parameter if compared to the energy values and elements of antisymmetric $3 \times 3$ real matrices and $\delta_{\mu \nu}$ is the identity matrix. The symbol $*$ denotes the Weyl Moyal star product, which is generalized between two ordinary functions $A(x) B(x)$ to the new deformed form $\hat{A}\left(x_{n c}\right) \hat{B}\left(x_{n c}\right)$ which expressed with the Weyl Moyal star product $A(x) * B(x)$ in the symmetries of NCQM as follows [37-43]:

$$
\begin{aligned}
(A * B)(x) & =\exp \left(i \epsilon^{\mu \nu} \theta \partial_{\mu}^{x} \partial_{\mu}^{x}\right)(A B)(x) \approx(f h)(x) \\
& \left.-\frac{i \epsilon^{\mu \nu} \theta}{2} \partial_{\mu}^{x} A \partial_{\mu}^{x} B\right\rfloor_{x^{\mu}=x^{\nu}}+O\left(\theta^{2}\right) .
\end{aligned}
$$

The indices $(\mu, \nu=1,2,3)$ and $O\left(\theta^{2}\right)$ stand for the second and higher-order terms of the NC parameter. Physically, the second term in Eq. (4) presents the effects of spacespace noncommutativity. Furthermore, it is possible to unify the operators $\widehat{X}_{\mu}^{H}(t)=\left(x_{n c \mu}^{H} \vee p_{n c \mu}^{H}\right)(t)$ and $\widehat{X}_{\mu}^{I}(t)=$ $\left(x_{n c \mu}^{I} \vee p_{n c \mu}^{I}\right)(t)$ in the Heisenberg and the interaction pictures using the following projection relations, respectively:

$$
\begin{gathered}
\underbrace{X_{\mu}^{H}(t)=\exp \left(i \widehat{H}_{r n c}^{\mathrm{hmp}} T\right) X_{\mu}^{S} \exp \left(-i \widehat{H}_{r n c}^{\mathrm{hmp} T}\right)}_{\text {QM-symmetry }} \\
\Rightarrow \underbrace{\widehat{X}_{\mu}^{H}(t)=\exp \left(i \widehat{H}_{r n c}^{\mathrm{hmp}} T\right) \widehat{X}_{\mu}^{S} \exp \left(-i \widehat{H}_{r n c}^{\mathrm{hmp}} T\right)}_{\text {NCQM-symmetry }},
\end{gathered}
$$

and

$$
\underbrace{X_{\mu}^{I}(t)=\exp \left(i \widehat{H}_{o r}^{\mathrm{hmp}} T\right) X_{\mu}^{S} \exp \left(-i \widehat{H}_{o r}^{\mathrm{hmp}} T\right)}_{\text {QM-symmetry }}
$$

$$
\Rightarrow \underbrace{\widehat{X}_{\mu}^{I}(t)=\exp \left(i \widehat{H}_{n c-o r}^{\mathrm{hmp}} T\right) \widehat{X}_{\mu}^{S} \exp \left(-i \widehat{H}_{n c-o r}^{\mathrm{hmp} T}\right)}_{\text {NCQM-symmetry }}
$$

Moreover, the dynamics of new systems $d \widehat{X}_{\mu}^{I}(t) / d t$ can be described by the following motion equations in the deformed Heisenberg picture as follows:

$$
\begin{aligned}
& \underbrace{\frac{d X_{\mu}^{H}(t)}{d t}=\left[X_{\mu}^{H}(t), \widehat{H}_{\mathrm{hmp}}\right]+\frac{\partial X_{\mu}^{H}(t)}{\partial t}}_{\text {QM-symmetry }} \\
& \underbrace{\frac{d \widehat{X}_{\mu}^{I}(t)}{d t}=\left[\widehat{X}_{\mu}^{I}(t) * \widehat{H}_{n c}^{\mathrm{hmp}}\right]+\frac{\partial \widehat{X}_{\mu}^{I}(t)}{\partial t}}_{\text {Extended QM-symmetry }} .
\end{aligned}
$$


Here ( $\widehat{H}_{o r}^{\mathrm{hmp}}$ and $\left.\widehat{H}_{n c-o r}^{\mathrm{hmp}}\right)$ are the free and total Hamiltonian operators for equal vector scalar of the Hellmann-generalized Morse potential in QM and EQM symmetries, while $\left(\widehat{H}_{\mathrm{hmp}}\right.$ and $\left.\widehat{H}_{n c}^{\mathrm{hmp}}\right)$ the Hamiltonians in QM and EQM symmetries. The purpose of this paper is to investigate the l-state solution of the deformed Klein-Gordon and Schrödinger equations within Bopp's shift and standard perturbation theory methods to generate an accurate new energy spectrum in ERQM and ENRQM symmetries. Our current work is structured in eight sections. The first one includes the scope and purpose of our investigation, while the remaining parts of the paper are structured as follows. A review of the Klein-Gordon, Dirac and Schrödinger equations with Hellmann-generalized Morse potential is presented in Sec. 2. Section 3 is devoted to studying the deformed Klein-Gordon equation by applying the ordinary Bopp's shift method and the Greene and Aldrich approximation for the centrifugal term to obtain the effective potential of the improved Hellmann-generalized Morse potential in RNCQM symmetries. Besides, via perturbation theory, we find the expectation values of some radial terms to calculate the energy shift produced by the effect of the perturbed effective potential of the improved Hellmann-generalized Morse potential. Section 4 is devoted to present the global energy shift and the global energy spectra produced by improved Hellmann-generalized Morse potential in the deformed Klein-Gordon symmetries. In Sec. 5, we examine some particular relativistic important cases in the context of the deformed Klein-Gordon theory. In the next section, we derive the global energy shift and the global energy spectra produced with improved Hellmann-generalized Morse potential in the deformed Dirac symmetries. In Sec. 7, we apply our study for determining the energy spectra of some selected diatomic molecules such as $\left(\mathrm{HCl}, \mathrm{LiH}, \mathrm{H}_{2}, \mathrm{ScH}, \mathrm{TiH}, \mathrm{VH}, \mathrm{CrH}, \mathrm{CuLi}\right.$, $\mathrm{TiC}, \mathrm{NiC}, \mathrm{ScN}$ and $\mathrm{ScF}$ ) in the ENRQM under improved Hellmann-generalized Morse potential, also, to study the composite systems. In Sec. 8, our conclusive remarks and future directions are given.

\section{Revised of Klein-Gordon, Dirac and Schrödinger equations under Hellmann-generalized Morse potential}

Before we start constructing the new solutions of the deformed Klein-Gordon, Dirac, and Schrödinger equations under the improved Hellmann-generalized Morse potential MGHPs, we give a summary of the corresponding usual solutions in ordinary relativistic quantum mechanics and nonrelativistic quantum mechanics. The Hellmann-generalized Morse potential in the symmetries of RQM and NRQM is given by [34,35]:

$$
V_{\mathrm{hmp}}(r)=D_{e}\left[1+\frac{-a+b e^{-\alpha r}}{r D_{e}}-2\left(\frac{e^{\alpha r_{e}}-1}{e^{\alpha r}-1}\right)+\left(\frac{e^{\alpha r_{e}}-1}{e^{\alpha r}-1}\right)^{2}\right] \text {, }
$$

and

$$
S_{\mathrm{hmp}}(r)=S_{e}\left[1+\frac{-a+b e^{-\alpha r}}{r S_{e}}-2\left(\frac{e^{\alpha r_{e}}-1}{e^{\alpha r}-1}\right)+\left(\frac{e^{\alpha r_{e}}-1}{e^{\alpha r}-1}\right)^{2}\right] .
$$

The 3-dimensional Klein-Gordon equation, Dirac equation with a scalar potential $S_{\mathrm{hmp}}(r)$ and a vector potential $V_{\mathrm{hmp}}(r)$, and the Schrödinger equation with the vector potential $V_{\mathrm{hmp}}(r)$ for the diatomic molecule (or fermionic particles) with reduced mass $M$ and wave function $\Psi_{n k}(r, \Omega)$ are given as:

$$
\begin{aligned}
\left(-\Delta+\left(M+S_{\mathrm{hmp}}(r)\right)^{2}-\left(E_{n l}-V_{\mathrm{hmp}}(r)\right)^{2}\right) \Psi(r, \Omega) & =0, \\
\left(\alpha p+\beta\left(M+S_{\mathrm{hmp}}(r)\right)\right) \Psi_{n k}(r, \Omega) & =\left(E_{n k}-V_{\mathrm{hmp}}(r)\right) \Psi_{n k}(r, \Omega), \\
\left(-\frac{\Delta}{2 M}+V_{\mathrm{hmp}}(r)\right) \Psi(r, \Omega) & =E_{n l}^{n r} \Psi(r, \Omega) .
\end{aligned}
$$

Here $\Delta$ is the ordinary 3-dimensional Laplacian operator, the vector potential $V_{\mathrm{hmp}}(r)$ due to the four-vector linear momentum operator $A^{\mu}\left(V_{\mathrm{hmp}}(r), \mathbf{A}=\mathbf{0}\right)$ and space-time scalar potential $S_{\mathrm{hmp}}(r)$ due to the mass, $\left(E_{n l}, E_{n k}\right)$ and $E_{n l}^{n r}$ are represents the relativistic and nonrelativistic energy eigenvalues, $(n, l)$ are the principal and orbital quantum numbers, respectively.

$$
\alpha_{i}=\left(\begin{array}{cc}
0 & \sigma_{i} \\
\sigma_{i} & 0
\end{array}\right), \quad \beta=\left(\begin{array}{cc}
I_{2 \times 2} & 0 \\
0 & I_{2 \times 2}
\end{array}\right),
$$

and $\sigma_{i}$ are the usual Pauli matrices. Since the Hellmann-generalized Morse potential has spherical symmetry, allowing the solutions of the time-independent Klein-Gordon equation and Schrödinger equation of the known form $\Psi(r, \Omega)=\left(\psi_{n l}(r) / r\right) Y_{l}^{m}(\Omega)$ to separate the radial $\psi_{n l}(r)$ and angular parts $Y_{l}^{m}(\Omega)$ of the wave function $\Psi(r, \Omega)$. For the Dirac equation,

$$
\Psi_{n k}(r, \Omega)=\frac{1}{r}\left(\begin{array}{c}
F_{n k}(r) Y_{j m}^{l}(\Omega) \\
i G_{n \widetilde{k}}(r) Y_{j m}^{\widetilde{l}}(\Omega)
\end{array}\right),
$$


where $F_{n k}(r)$ and $G_{n k}(r)$ represent the upper and lower components of the Dirac spinors $\Psi_{n k}(r, \Omega)$ while $Y_{j m}^{l}(\Omega)$ and $Y_{j m}^{\tilde{l}}(\Omega)$ are the spin and pseudospin spherical harmonics and $m$ is the projection on the z-axis. Thus, Eqs. (10.1), (10.2) and (10.3) can be expressed as:

$$
\begin{aligned}
& \left(\frac{d^{2}}{d r^{2}}-\left[M^{2}-E_{n l}^{2}\right]-2\left[E_{n l} V_{\mathrm{hmp}}\{r\}+M S_{\mathrm{hmp}}\{r\}\right]+V_{\mathrm{hmp}}^{2}[r]-S_{\mathrm{hmp}}^{2}[r]-\frac{l[l+1]}{r^{2}}\right) \psi_{n l}(r)=0, \\
& \left(\frac{d^{2}}{d r^{2}}-\frac{k[k+1]}{r^{2}}-\left[M+E_{n k}-\Delta\{r\}\right]\left[M-E_{n k}+\Sigma\{r\}\right]+\frac{\frac{d \Delta(r)}{d r}\left[\frac{d}{d r}+\frac{k}{r}-\psi\{r\}\right]}{\left[M+E_{n k}-\Delta\{r\}\right]}\right) F_{n k}(r)=0, \\
& \left(\frac{d^{2}}{d r^{2}}-\frac{k[k-1]}{r^{2}}-\left[M+E_{n k}-\Delta\{r\}\right]\left[M-E_{n k}+\Sigma\{r\}\right]+\frac{\frac{d \Sigma(r)}{d r}\left[\frac{d}{d r}-\frac{k}{r}+\psi\{r\}\right]}{\left(M+E_{n k}-\Sigma(r)\right)}\right) G_{n \widetilde{k}}(r)=0, \\
& \left(\frac{d^{2}}{d r^{2}}+2 M\left[E_{n l}^{n r}-V_{\mathrm{hmp}}\{r\}-\frac{l\{l+1\}}{2 M r^{2}}\right]\right) \psi_{n l}(r)=0 .
\end{aligned}
$$

Using the shorthand notation $E_{e f f}^{\mathrm{hmp}}=M^{2}-E_{n l}^{2}$ and:

$$
\begin{aligned}
& V_{e f f}^{\mathrm{hmp}}(r)=2\left(E_{n l} V_{\mathrm{hmp}}(r)+M S_{\mathrm{hmp}}(r)\right)-V_{\mathrm{hmp}}^{2}(r)+S_{\mathrm{hmp}}^{2}(r)+\frac{l(l+1)}{r^{2}}, \\
& \sum(r)=D_{e} 1+\frac{-a+b e^{-\alpha r}}{r D_{e}}-2\left(\frac{e^{\alpha r_{e}}-1}{e^{\alpha r}-1}\right)+\left(\frac{e^{\alpha r_{e}}-1}{e^{\alpha r}-1}\right)^{2} \quad \text { and } \quad \frac{d \Delta(r)}{d r}=0 \Longrightarrow \Delta(r)=C_{s} \\
& \text { For Spin Symmetry Limit } \\
& \Delta(r)=D_{e}\left[1+\frac{-a+b e^{-\alpha r}}{r D_{e}}-2\left(\frac{e^{\alpha r} e-1}{e^{\alpha r}-1}\right)+\left(\frac{e^{\alpha r_{e}}-1}{e^{\alpha r}-1}\right)^{2}\right] \quad \text { and } \quad \frac{d \Sigma(r)}{d r}=0 \Longrightarrow \Sigma(r)=C_{p s} \\
& \text { For Pseudospin Symmetry Limit } \\
& V_{e f f-n r}^{\mathrm{hmp}}(r)=V_{\mathrm{hmp}}(r)+\frac{l(l+1)}{r^{2}} .
\end{aligned}
$$

We obtain the following second-order Schrödinger-like equation in RQM and NRQM symmetries, respectively:

$$
\begin{array}{r}
\left(\frac{d^{2}}{d r^{2}}-\left[E_{e f f}^{\mathrm{hmp}}+V_{e f f}^{\mathrm{hmp}}(r)\right]\right) \psi_{n l}(r)=0, \\
\left(\frac{d^{2}}{d r^{2}}-\frac{k(k+1)}{r^{2}}-\left[M+E_{n k}^{s}-C_{s}\right]\left[M-E_{n k}^{s}-\frac{a}{r}+\frac{b e^{-\alpha r}}{r}+D_{e}\left\{1+\frac{q}{e^{-\alpha r}-1}\right\}^{2}\right]\right) F_{n k}(r)=0, \\
\left(\frac{d^{2}}{d r^{2}}-\frac{k(k-1)}{r^{2}}-\left[M-E_{n k}^{p s}+\Sigma_{c}\right]\left[M+E_{n k}^{p s}+\frac{a}{r}-\frac{b e^{-\alpha r}}{r}-D_{e}\left\{1-\frac{q}{e^{-\alpha r}-1}\right\}^{2}\right]\right) G_{n \tilde{k}}(r)=0, \\
\left(\frac{d^{2}}{d r^{2}}+2 M\left[E_{n l}^{n r}-V_{e f f-n r}^{\mathrm{hmp}}\{r\}\right]\right) \psi_{n l}(r)=0 .
\end{array}
$$

With $k(k-1)=\widetilde{l}(\widetilde{l}-1)$ and $k(k+1)=l(l+1)$. When the vector potential is equal to the scalar potential $V_{m p}(r)=$ $S_{m p}(r)$ the effective potential leads to the following simple form

$$
V_{e f f}^{\mathrm{hmp}}(r)=2\left(E_{n l}+M\right) V_{\mathrm{hmp}}(r)+\frac{l(l+1)}{r^{2}} .
$$

The authors of Refs. [34, 35] using both Nikiforov-Uvarov method and the Greene and Aldrich approximation for the centrifugal term to obtain the expressions for the wave function as hypergeometric polynomials and the corresponding energy 
values for improved Hellmann-generalized Morse potential, in RQM and NRQM symmetries as,

$$
\begin{aligned}
\Psi(r, \Omega) & =\frac{n ! \Gamma\left(n+A_{n l}+1\right) N_{n l}}{\Gamma\left(2 A_{n l}+1\right)} s^{A_{n l} / 2}(1-s)^{V_{n l}+\frac{1}{2}}{ }_{2} F_{1}\left(-n, n+V_{n l}+A_{n l}+1 ; 1+A_{n l} ; s\right) Y_{l}^{m}(\Omega), \\
F_{n k}(r) & =\frac{N_{n k}\left(2 \omega_{n k}+1\right)_{n}}{n !} s^{\omega_{n k}}(1-s)^{\lambda_{n k}+\frac{1}{2}}{ }_{2} F_{1}\left(-n, n+2 \omega_{n k}+2 \lambda_{n k}+1 ; 1+\omega_{n k} ; s\right) Y_{l}^{m}(\Omega), \\
G_{n \widetilde{k}}(r) & =\frac{N_{n k}\left(2 \Omega_{n k}+1\right)_{n}}{n !} s^{\Omega_{n k}}(1-s)^{\beta_{n l}+\frac{1}{2}}{ }_{2} F_{1}\left(-n, n+2 \Omega_{n k}+2 \beta_{n l}+1 ; 1+\Omega_{n k} ; s\right) Y_{l}^{m}(\Omega),
\end{aligned}
$$

and

$$
\begin{aligned}
E_{n l}^{2}-M^{2} & =\left(D_{e}-\alpha a\right)\left(E_{n l}+M\right)+\alpha^{2} l(l+1)-\frac{1}{4}\left(\frac{\alpha\left[n+\frac{1}{2}+\delta_{n l}\right]-\left[E_{n l}+M\right]\left[a-b+2 \frac{D_{e}}{\alpha}\left\{e^{\alpha r_{e}}-1\right\}\right]}{n+\frac{1}{2}+l[l+1]}\right. \\
& \left.-\frac{\left[E_{n l}+M\right]\left[\frac{D_{e}}{\alpha}\left\{e^{\alpha r_{e}}-1\right\}^{2}+\alpha l\{l+1\}\right]}{n+\frac{1}{2}+l[l+1]}\right)^{2} \\
E_{n l}^{n r} & =D_{e}-\alpha a+\frac{\alpha^{2} l(l+1)}{2 M}-\frac{\alpha^{2}}{8 M}\left(\frac{\Lambda(n, l)-2 M \eta(l)}{\Lambda(n, l)}\right)^{2} .
\end{aligned}
$$

For the spin symmetry, the equation of energy is given by:

$$
\begin{aligned}
\left(M+E_{n k}^{s p}-C_{s}\right)\left(M-E_{n k}^{s p}+D_{e}\right) & =\left(M+E_{n k}^{s p}-C_{s}\right) \alpha a-\alpha^{2} k(k+1) \\
& +\frac{\alpha^{2}}{4}\left(\frac{\Lambda_{s}^{2}[n, k]+\left[M-E_{n k}^{s p}+D_{e}\right] \eta_{s}[k]}{\Lambda_{s}[n, k]}\right)^{2} .
\end{aligned}
$$

For the pseudospin symmetry, the equation of energy is given by:

$$
\begin{aligned}
\left(D_{e}-M-E_{n k}^{p s}\right)\left(M-E_{n k}^{p s}-C_{p s}\right) & =\left(M-E_{n k}^{p s}-C_{p s}\right) \alpha a-\alpha^{2} k(k-1) \\
& +\frac{\alpha^{2}}{4}\left(\frac{\Lambda_{s}^{2}[n, k]+\left[M-E_{n k}^{p s}+D_{e}\right] \eta_{s}[k]}{\Lambda_{s}[n, k]}\right)^{2},
\end{aligned}
$$

with

$$
\begin{aligned}
\delta_{n l} & =\sqrt{\frac{1}{4}+\frac{D_{e}}{\alpha^{2}}\left(E_{n l}+M\right)\left(e^{\alpha r_{e}}-1\right)^{2}+\alpha l(l+1)}, \\
A_{n l} & =\sqrt{\epsilon_{n l}-\frac{a}{\alpha^{2}}\left(E_{n l}+M\right)+4 l(l+1)}, \\
V_{n l} & =\sqrt{\epsilon_{n l}+\frac{D_{e} q^{2}}{\alpha^{2}}\left(E_{n l}+M\right)+4 l(l+1)}, \\
\omega_{n k} & =\sqrt{\frac{\left(M+E_{n k}^{s p}-C_{s}\right)\left(M-E_{n k}^{s p}+D_{e}\right)}{\alpha^{2}}-\frac{\left(E_{n k}^{s p}+M\right) a}{\alpha}+k(k+1)}, \\
\Omega_{n k} & =\sqrt{\frac{\left(M+E_{n k}^{p s}-D_{e}\right)\left(M-E_{n k}^{p s}+C_{p s}\right)}{\alpha^{2}}-\frac{\left(E_{n k}^{p s}+M+C_{p s}\right) a}{\alpha}+k(k-1)}, \\
\beta_{n k} & =\sqrt{\frac{1}{4}+\frac{D_{e}}{\alpha^{2}}\left(M-E_{n k}^{p s}+C_{p s}\right) D_{e} q^{2}+l(l+1)} \\
\eta_{l} & =\frac{b}{\alpha}-\frac{2 D_{e} q^{2}}{\alpha^{2}}-\frac{\alpha}{\alpha}+\frac{2 D_{e} q^{2}}{\alpha^{2}}+l(l+1),
\end{aligned}
$$




$$
\begin{aligned}
\Lambda_{n l} & =n+\frac{1}{2}+\sqrt{\frac{1}{4}+\frac{D_{e} q^{2}}{\alpha^{2}}+\alpha l(l+1)}, \\
\Lambda_{s}(n, k) & =n+\frac{1}{2}+\sqrt{\frac{1}{4}+\frac{D_{e}}{\alpha^{2}}\left(M+E_{n k}^{s p}-C_{s}\right)+k(k+1)}, \\
\Lambda_{p s}(n, k) & =n+\frac{1}{2}+\sqrt{\frac{1}{4}+\frac{D_{e}}{\alpha^{2}}\left(M+E_{n k}^{p s}-C_{p s}\right)+k(k-1)},
\end{aligned}
$$

and

$$
\begin{aligned}
& \eta_{s}(k)=\frac{b}{\alpha}-\frac{2 D_{e} q^{2}}{\alpha^{2}}-\left(\frac{\alpha}{\alpha}\right)^{2}+\frac{2 D_{e} q^{2}}{\alpha^{2}}+k(k+1), \\
& \eta_{p}(k)=\frac{b}{\alpha}-\frac{2 D_{e} q^{2}}{\alpha^{2}}-\left(\frac{\alpha}{\alpha}\right)^{2}+\frac{2 D_{e} q^{2}}{\alpha^{2}}+k(k-1) .
\end{aligned}
$$

The Pochhammer symbol is $(m)_{n}=\Gamma[m+n] / \Gamma[m+n]$, and $s=e^{-\alpha r}, N_{n l}^{m 2}$ and $N_{n k l}$ are the normalization constants, (see Refs. [34,35]).

\section{The new solutions of DKGE under IHGMP in the EQM}

\subsection{Review of Bopp's shift method}

Let us begin in this subsection by finding the deformed Klein Gordon equation (DKGE) in the symmetries of relativistic noncommutative quantum mechanics or the extended quantum mechanics under the improved Hellmann-generalized Morse potential (IHGMP). Our goal is achieved by applying the new principles which we have seen in the introduction, Eqs. (2.1), (2.2) and (3), summarized in new relations and the notion of the Weyl-Moyal star product. These data allow us to rewrite the usual radial Klein-Gordon equation in Eq. (13.1) in the ERQM symmetries as follows [54-57,76-85]:

$$
\left(\frac{d^{2}}{d r^{2}}-\left[E_{\mathrm{eff}}^{\mathrm{hmp}}+V_{\mathrm{eff}}^{\mathrm{hmp}}(r)\right]\right) * \psi_{n l}(r)=0 .
$$

It is established extensively in the literature that star products can be simplified by Bopp's shift method. The physicist Fritz Bopp was the first to consider pseudo-differential operators obtained from a symbol by the quantization rules $x \rightarrow x-$ $(i / 2)(\partial / \partial p)$, and $p \rightarrow p+(i / 2)(\partial / \partial x)$ instead of the ordinary correspondence $x \rightarrow x$ and $p \rightarrow(i / 2)(\partial / \partial x)$. This is known by Bopp's shifts and this quantization procedure is called Bopp quantization [85-87]. It is known to the specialists that Bopp's shift method has been applied effectively and has succeeded in simplifying the three basic equations: the deformed Klein-Gordon equation [54-57,76-85], deformed Dirac equation [88-91], deformed Schrödinger equation [92-95] and Duffin-Kemmer-Petiau equation [81,82] with the notion of star product to the Klein-Gordon equation, the Dirac equation and the Schrödinger equation with the notion of ordinary product. Thus, Bopp's shift method is based on reducing second order linear differential equations of the deformed Klein-Gordon equation, the deformed Dirac equation, and the deformed Schrödinger equation with star product to second-order linear differential equations of Klein-Gordon equation, Dirac equation, and Schrödinger equation without star product with simultaneous translation in the space-space. The CNCCRs with star product in Eqs. (2.1) and (2.2) become new CNCCRs without the notion of star product as follows (see, e.g., [54-57,76-85]):

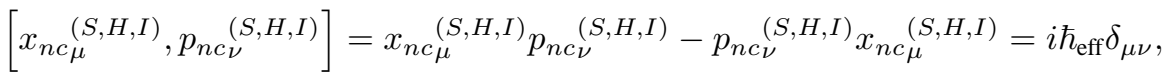

$$
\begin{aligned}
& {\left[x_{n c \mu}{ }^{(S, H, I)}, x_{n c \nu}{ }^{(S, H, I)}\right]=x_{n c \mu}{ }^{(S, H, I)} x_{n c \nu}{ }^{(S, H, I)}-x_{n c \nu}{ }^{(S, H, I)} x_{n c \mu}{ }^{(S, H, I)}=i \theta_{\mu \nu} .}
\end{aligned}
$$

The generalized positions and momentum coordinates $x_{n c \mu}^{(S, H, I)}=\left(x_{n c \mu}^{S}, x_{n c \mu}^{H}, x_{n c \mu}^{I}\right)$ and $p_{n c \mu}^{(S, H, I)}=\left(p_{n c \mu}^{S}, p_{n c \mu}^{H}, p_{n c \mu}^{I}\right)$, in the symmetries of ERQM are defined in terms of the corresponding coordinates in the symmetries of RQM $x_{\mu}^{(S, H, I)}=$ $\left(x_{\mu}^{S}, x_{\mu}^{H}, x_{\mu}^{I}\right)$ and $p_{\mu}^{(S, H, I)}=\left(p_{\mu}^{S}, p_{\mu}^{H}, p_{\mu}^{I}\right)$ via, respectively [54-57,76-85]:

$$
x_{n c}{ }_{\mu}^{(S, H, I)}=x_{\mu}^{(S, H, I)}-\sum_{\nu=1}^{3} \frac{i \theta_{\mu \nu}}{2} p_{\nu}^{(S, H, I)} \quad \text { and } \quad p_{n c_{\mu}}^{(S, H, I)}=p_{\mu}^{(S, H, I)} .
$$

This allows us to find the operator $r_{n c}^{2}$ equal $r^{2}-\mathbf{L} \Theta$ in NCQM symmetries [54-57,76-85]. New effective potential for MHGPs in ERQM symmetries 


\subsection{The new effective potential of the improved Hellmann-generalized Morse potential model in DRKGT symmetries}

According to the Bopp shift method, Eq. (19) with star product becomes similar to the following like the Schrödinger equation (without the notions of star product):

$$
\left(\begin{array}{c}
\frac{d^{2}}{d r^{2}}--\left(M^{2}-E_{n l}^{2}\right)-\frac{l(l+1)}{r_{n c}^{2}} \\
-V_{\mathrm{hmp}}\left(r_{n c}\right)\left(E_{n l}+M\right)
\end{array}\right) \psi_{n l}(r)=0 .
$$

The new operators $V_{\mathrm{hmp}}\left(r_{n c}\right)$ and $\left(l(l+1) / r_{n c}^{2}\right)$ are expressed as in ERQM symmetries as follows:

$$
V_{\mathrm{hmp}}\left(r_{n c}\right)=D_{e}-\frac{a}{r}+\frac{b e^{-\alpha r}}{r}-2 \frac{D_{e} q e^{-\alpha r}}{1-e^{-\alpha r}}+\frac{D_{e} q^{2} e^{-2 \alpha r}}{\left(1-e^{-\alpha r}\right)^{2}}-\frac{\partial V_{\mathrm{hmp}}(r)}{\partial r} \frac{\mathbf{L} \Theta}{2 r}+O\left(\Theta^{2}\right),
$$

and

$$
\frac{l(l+1)}{r_{n c}^{2}}=\frac{l(l+1)}{r^{2}}+\frac{l(l+1)}{r^{4}} \mathbf{L} \Theta+O\left(\Theta^{2}\right) .
$$

Here $q=e^{\alpha r_{e}}-1$. Therefore, we can rewrite:

$$
2 V_{\mathrm{hmp}}\left(r_{n c}\right)\left(E_{n l}+M\right)=2 V_{\mathrm{hmp}}(r)\left(E_{n l}+M\right)-\left(\frac{E_{n l}+M}{r}\right) \frac{\partial V_{\mathrm{hmp}}(r)}{\partial r} \mathbf{L} \Theta+O\left(\Theta^{2}\right) .
$$

Moreover, to illustrate the above equation in a simple mathematical way and attractive form, it is useful to enter the following symbol $V_{n c-\text { eff }}^{\mathrm{hmp}}(r)$, thus the radial Eq. (22) becomes:

$$
\left(\frac{d^{2}}{d r^{2}}-\left[E_{\mathrm{eff}}^{\mathrm{hmp}}+V_{n c-\mathrm{eff}}^{\mathrm{hmp}}(r)\right]\right) \psi_{n l}(r)=0
$$

with:

$$
V_{n c-e f f}^{\mathrm{hmp}}(r)=V_{\mathrm{eff}}^{\mathrm{hmp}}(r)+V_{\text {pert }}^{\mathrm{hmp}}(r) .
$$

Moreover, $V_{\text {pert }}^{\mathrm{hmp}}(r)$ is given by the following relation:

$$
V_{p e r t}^{\mathrm{hmp}}(r)=\left(\frac{l(l+1)}{r^{4}}-\frac{E_{n l}+M}{r} \frac{\partial V_{\mathrm{hmp}}(r)}{\partial r}\right) \mathbf{L} \Theta+O\left(\Theta^{2}\right) .
$$

It should be noted that when $l=0$ the Eq. (13.1) can be exactly solved analytically for the case $l \neq 0$, the authors of Refs. [34,34] approximatively solved the equation using the Greene and Aldrich approximation in relativistic and nonrelativistic quantum mechanics symmetries. In the new form of radial like-Schrödinger equation written in Eq. (26), we have observed new terms including $\left((1 / r),\left(1 / r^{4}\right)\right.$ and other Columbia-like terms) which make this equation impossible to solve analytically for and, it can only be solved approximately. From this point of view, we can consider the Greene and Aldrich approximation [96]. It is also used in many other works [97-99]:

$$
\frac{1}{r^{2}} \approx \frac{\alpha^{2}}{\left(1-e^{-2 \alpha r}\right)^{2}}=\frac{\alpha^{2}}{(1-s)^{2}}
$$

It is important to mention here that the above approximations are valid in short when $\alpha r \ll 1$. This allows us to obtain:

$$
\frac{1}{r} \approx \frac{\alpha}{1-e^{-2 \alpha r}}=\frac{\alpha}{1-s} .
$$

After straightforward calculations $\left(\partial V_{\mathrm{hmp}}(r) / \partial r\right)$ we obtain as follows:

$$
\frac{\partial V_{\mathrm{hmp}}(r)}{\partial r}=\frac{a}{r^{2}}-\frac{b \alpha e^{-\alpha r}}{r}-\frac{b \alpha e^{-\alpha r}}{r^{2}}-2 \frac{D_{e} \alpha q e^{-\alpha r}}{1-e^{-\alpha r}}+\frac{D_{e} q(1-q) e^{-2 \alpha r}}{\left(1-e^{-\alpha r}\right)^{2}}-2 \frac{D_{e} \alpha q^{2} e^{-3 \alpha r}}{\left(1-e^{-\alpha r}\right)^{3}} .
$$

Upon invoking the Greene-Aldrich approximation scheme, the expression $\left(E_{n l}+M / r\right)\left(\partial V_{\mathrm{hmp}}(r) / \partial r\right)$ reduces to the form:

$$
\frac{E_{n l}+M}{r} \frac{\partial V_{\mathrm{hmp}}(r)}{\partial r}=\alpha\left(E_{n l}+M\right)\left(\frac{a}{(1-s)^{3}}-\frac{b \alpha s}{(1-s)^{3}}+\left(2 D_{e} q-b\right) \frac{\alpha s}{(1-s)^{2}}+2 \frac{D_{e} \alpha q(1-q) s^{2}}{(1-s)^{3}}-2 \frac{D_{e} \alpha q^{2} s^{3}}{(1-s)^{4}}\right) .
$$


By making the substitution Eq. (32) into Eq. (28), we find the perturbed effective potential $V_{\text {pert }}^{\text {hmp }}(s)$ generated from noncommutativity properties of space-space that produced by the effect of IHGMP in the symmetries of ERQM as follows:

$$
\begin{aligned}
V_{\text {pert }}^{\mathrm{hmp}}(s) & =\left(\frac{l(l+1) \alpha^{4}}{(1-s)^{4}}-\alpha\left(E_{n l}+M\right)\left[\frac{a}{(1-s)^{3}}-\frac{b \alpha s}{(1-s)^{3}}\right.\right. \\
& \left.\left.+\left(2 D_{e} q-b\right) \frac{\alpha s}{(1-s)^{2}}+2 \frac{D_{e} \alpha q(1-q) s^{2}}{(1-s)^{3}}-2 \frac{D_{e} \alpha q^{2} s^{3}}{(1-s)^{4}}\right]\right) \mathbf{L} \Theta+O\left(\Theta^{2}\right) .
\end{aligned}
$$

We have replaced the term $\left(l[l+1] / r^{4}\right)$ with the Greene and Aldrich approximation in Eq. (28). The equal scalar and vector improved Hellmann-generalized Morse potentials are extended by including new terms proportional to the radial terms $\left(1 /[1-s]^{4}\right),\left(1 /[1-s]^{3}\right),\left(s /[1-s]^{3},\left(s /[1-s]^{2}\right),\left(s^{2} /[1-s]^{3}\right)\right.$ and $\left(s^{3} /[1-s]^{4}\right)$ become the improved Hellmanngeneralized Morse potential in ERQM symmetries. The generated new effective potential $V_{\text {pert }}^{\mathrm{hmp}}(s)$ is also proportional to the infinitesimal vector $\boldsymbol{\Theta}$. This allows us to consider the new additive part of the effective potential $V_{\text {pert }}^{\text {hmp }}(s)$ as a perturbation potential compared with the main potential $V_{e f f}^{\mathrm{hmp}}(s)$ (the parent potential operator in the symmetries of ERQM, that is, the inequality has become achieved $V_{\text {pert }}^{\mathrm{hmp}}(s) \ll V_{\mathrm{eff}}^{\mathrm{hmp}}(s)$. That is all physical justifications for applying the time-independent perturbation theory become satisfied. This allows us to give a complete prescription for determining the energy level of the generalized $n^{\text {th }}$ excited states.

\subsection{The expectation values under IHGMP in the deformed Klein-Gordon symmetries}

In this subsection, we want to apply the perturbative theory, in the case of ERQM symmetries, we find the expectation values of the radial terms $\left(1 /[1-s]^{4}\right),\left(1 /[1-s]^{3}\right),\left(s /[1-s]^{3},\left(s /[1-s]^{2}\right),\left(s^{2} /[1-s]^{3}\right)\right.$ and $\left(s^{3} /[1-s]^{4}\right)$ taking into account the wave function which we have seen previously in Eq. (15.1). Thus after straightforward calculations, we obtain the following results:

$$
\begin{aligned}
& \left.\left\langle\frac{1}{(1-s)^{4}}\right\rangle_{(n l m)}=N_{n l}^{m 2} \int_{0}^{+\infty} \frac{s^{2}}{(1-s)^{4}} s^{A_{n l}}(1-s)^{2 V_{n l}+1}{ }_{2} F_{1}\left(-n, n+V_{n l}+A_{n l}+1 ; 1+A_{n l} ; s\right)\right]^{2} d r \\
& \left\langle\frac{1}{(1-s)^{3}}\right\rangle_{(n l m)}=N_{n l}^{m 2} \int_{0}^{+\infty} \frac{1}{(1-s)^{3}} s^{A_{n l}}(1-s)^{2 V_{n l}+1}\left[{ }_{2} F_{1}\left(-n, n+V_{n l}+A_{n l}+1 ; 1+A_{n l} ; s\right)\right]^{2} d r \\
& \left\langle\frac{s}{(1-s)^{3}}\right\rangle_{(n l m)}=N_{n l}^{m 2} \int_{0}^{+\infty} \frac{s}{(1-s)^{3}} s^{A_{n l}}(1-s)^{2 V_{n l}+1}\left[{ }_{2} F_{1}\left(-n, n+V_{n l}+A_{n l}+1 ; 1+A_{n l} ; s\right)\right]^{2} d r \\
& \left\langle\frac{s}{(1-s)^{2}}\right\rangle_{(n l m)}=N_{n l}^{m 2} \int_{0}^{+\infty} \frac{s}{(1-s)^{2}} s^{A_{n l}}(1-s)^{2 V_{n l}+1}\left[{ }_{2} F_{1}\left(-n, n+V_{n l}+A_{n l}+1 ; 1+A_{n l} ; s\right)\right]^{2} d r \\
& \left\langle\frac{s^{2}}{(1-s)^{3}}\right\rangle=N_{n l}^{m 2} \int_{0}^{+\infty} \frac{s^{2}}{(1-s)^{3}} s^{A_{n l}}(1-s)^{2 V_{n l}+1}\left[{ }_{2} F_{1}\left(-n, n+V_{n l}+A_{n l}+1 ; 1+A_{n l} ; s\right)\right]^{2} d r, \\
& \left\langle\frac{s^{3}}{(1-s)^{4}}\right\rangle=N_{n l}^{m 2} \int_{0}^{+\infty} \frac{s^{3}}{(1-s)^{4}} s^{A_{n l}}(1-s)^{2 V_{n l}+1}\left[{ }_{2} F_{1}\left(-n, n+V_{n l}+A_{n l}+1 ; 1+A_{n l} ; s\right)\right]^{2} d r,
\end{aligned}
$$

with $N_{n l}^{m 2}=\left(n ! \Gamma\left[n+A_{n l}+1\right] N_{n l} / \Gamma\left[2 A_{n l}+1\right]\right)^{2}$ and we have used useful abbreviations $\langle X\rangle_{(n l m)}=\langle n, l, m X n, l, m\rangle$ to avoid the extra burden of writing equations. Furthermore, we have applied the property of the spherical harmonics, which has the form $\int Y_{l}^{m}\left(\Omega^{\prime}\right) Y_{l^{\prime}}^{m^{\prime}}(\Omega) d^{2} \Omega=\delta_{l l^{\prime}} \delta_{m m^{\prime}}$. We have $s=e^{-\alpha r}$, this allows us to obtain $d r=-(1 / \alpha)(d s / s)$. From the asymptotic behavior of $s=e^{-\alpha r}$ when $(r \rightarrow 0)(s \rightarrow+1)$ and when $(r \rightarrow+\infty)(s \rightarrow 0)$, this allows to reformulate Eqs. $(34, i=\overline{1,6})$ as follows: 


$$
\begin{aligned}
& \left\langle\frac{1}{(1-s)^{4}}\right\rangle_{(n l m)}=\frac{N_{n l}^{m 2}}{\alpha} \int_{0}^{+1} s^{A_{n l}-1}(1-s)^{2 V_{n l}-3}\left[{ }_{2} F_{1}\left(-n, n+A_{n l}+V_{n l}+1 ; 1+A_{n l}, s\right)\right]^{2} d s, \\
& \left\langle\frac{1}{(1-s)^{3}}\right\rangle_{(n l m)}=\frac{N_{n l}^{m 2}}{\alpha} \int_{0}^{+1} s^{A_{n l}-1}(1-s)^{2 V_{n l}-2}\left[{ }_{2} F_{1}\left(-n, n+A_{n l}+V_{n l}+1 ; 1+A_{n l}, s\right)\right]^{2} d s, \\
& \left\langle\frac{s}{(1-s)^{3}}\right\rangle_{(n l m)}=\frac{N_{n l}^{m 2}}{\alpha} \int_{0}^{+1} s^{A_{n l}}(1-s)^{2 V_{n l}-2}\left[{ }_{2} F_{1}\left(-n, n+A_{n l}+V_{n l}+1 ; 1+A_{n l}, s\right)\right]^{2} d s, \\
& \left\langle\frac{s}{(1-s)^{2}}\right\rangle_{(n l m)}=\frac{N_{n l}^{m 2}}{\alpha} \int_{0}^{+1} s^{A_{n l}}(1-s)^{2 V_{n l}-1}\left[{ }_{2} F_{1}\left(-n, n+A_{n l}+V_{n l}+1 ; 1+A_{n l}, s\right)\right]^{2} d s, \\
& \left\langle\frac{s^{2}}{(1-s)^{3}}\right\rangle=\frac{N_{n l}^{m 2}}{\alpha} \int_{0}^{+1} s^{A_{n l}+1}(1-s)^{2 V_{n l}-2}\left[{ }_{2} F_{1}\left(-n, n+A_{n l}+V_{n l}+1 ; 1+A_{n l}, s\right)\right]^{2} d s, \\
& \left\langle\frac{s^{3}}{(1-s)^{4}}\right\rangle=\frac{N_{n l}^{m 2}}{\alpha} \int_{0}^{+1} s^{A_{n l}+2}(1-s)^{2 V_{n l}-3}\left[{ }_{2} F_{1}\left(-n, n+A_{n l}+V_{n l}+1 ; 1+A_{n l}, s\right)\right]^{2} d s .
\end{aligned}
$$

We can use the method proposed by Dong et al. [100] and applied by Zhang [101], we calculate the integrals in Eqs. $(35, i=$ $\overline{1,6})$. With the help of the special integral formula

$$
\int_{0}^{+1} s^{\xi-1}(1-s)^{\sigma-1}\left[{ }_{2} F_{1}\left(c_{1}, c_{2} ; c_{3} ; s\right)\right]^{2} d s=\frac{\Gamma(\xi) \Gamma(\sigma)}{\Gamma(\xi+\sigma)}{ }_{3} F_{2}\left(c_{1}, c_{2}, \sigma ; c_{3}, \sigma+\xi ; 1\right),
$$

here ${ }_{2} F_{1}\left(c_{1}, c_{2} ; c_{3} ; s\right)$ is the generalized hypergeometric function:

$$
{ }_{3} F_{2}\left(c_{1}, c_{2}, \sigma ; c_{3}, \sigma+\xi ; 1\right)=\sum_{n=0}^{+\infty} \frac{\left(c_{1}\right)_{n}\left(c_{2}\right)_{n}(\sigma)_{n}}{\left(c_{3}\right)_{n}(\sigma+\xi) n !} .
$$

Is obtained from the generalized hypergeometric function which has parameters $p$ of type 1 and $q$ parameters of type 2 of the form the following formula:

$$
{ }_{p} F_{q}\left(\alpha_{1}, \alpha_{2}, \ldots, \alpha_{p} ; \beta_{1}, \beta_{2}, \ldots, \beta_{1} ; 1\right)=\sum_{n=0}^{+\infty} \frac{\left(\alpha_{1}\right)_{n} \ldots\left(\alpha_{p}\right)_{n}}{\left(\beta_{1}\right)_{n} \ldots\left(\beta_{p}\right)_{n} n !},
$$

for $p=3$ and $1=2$ while $\Gamma(\sigma)$ denoting the usual Gamma function. We obtain from Eqs. $(35, i=\overline{1,6})$ the following results:

$$
\begin{aligned}
& \left\langle\frac{1}{(1-s)^{4}}\right\rangle_{(n l m)}=N_{n l}^{v i r} \frac{\Gamma\left(A_{n l}\right) \Gamma\left(2 V_{n l}-2\right)}{\Gamma\left(K_{n l}-2\right)}{ }_{3} F_{2}\left(-n, n+A_{n l}+V_{n l}+1,2 V_{n l}-2 ; 1+A_{n l}, K_{n l}-2 ; 1\right), \\
& \left\langle\frac{1}{(1-s)^{3}}\right\rangle_{(n l m)}=N_{n l}^{v i r} \frac{\Gamma\left(A_{n l}\right) \Gamma\left(2 V_{n l}-1\right)}{\Gamma\left(K_{n l}-1\right)}{ }_{3} F_{2}\left(-n, n+A_{n l}+V_{n l}+1,2 V_{n l}-1 ; 1+A_{n l}, K_{n l}-1 ; 1\right), \\
& \left\langle\frac{s}{(1-s)^{3}}\right\rangle_{(n l m)}=N_{n l}^{v i r} \frac{\Gamma\left(A_{n l}+1\right) \Gamma\left(2 V_{n l}-1\right)}{\Gamma\left(K_{n l}\right)}{ }_{3} F_{2}\left(-n, n+A_{n l}+V_{n l}+1,2 V_{n l}-1 ; 1+A_{n l}, K_{n l} ; 1\right),
\end{aligned}
$$




$$
\begin{aligned}
& \left\langle\frac{s}{(1-s)^{2}}\right\rangle_{(n l m)}=N_{n l}^{v i r} \frac{\Gamma\left(A_{n l}+1\right) \Gamma\left(2 V_{n l}\right)}{\Gamma\left(K_{n l}+1\right)}{ }_{3} F_{2}\left(-n, n+A_{n l}+V_{n l}+1,2 V_{n l}-1 ; 1+A_{n l}, K_{n l}+1 ; 1\right), \\
& \left\langle\frac{s^{2}}{(1-s)^{-3}}\right\rangle_{(n l m)}=N_{n l}^{v i r} \frac{\Gamma\left(A_{n l}+2\right) \Gamma\left(2 V_{n l}-1\right)}{\Gamma\left(K_{n l}+1\right)}{ }_{3} F_{2}\left(-n, n+A_{n l}+V_{n l}+1,2 V_{n l}-1 ; 1+A_{n l}, K_{n l}+1 ; 1\right), \\
& \left\langle\frac{s^{3}}{(1-s)^{4}}\right\rangle_{(n l m)}=N_{n l}^{v i r} \frac{\Gamma\left(A_{n l}+3\right) \Gamma\left(2 V_{n l}-2\right)}{\Gamma\left(K_{n l}+1\right)}{ }_{3} F_{2}\left(-n, n+A_{n l}+V_{n l}+1,2 V_{n l}-1 ; 1+A_{n l}, K_{n l}+1 ; 1\right),
\end{aligned}
$$

with $N_{n l}^{v i r}=\left(n ! \Gamma\left(n+A_{n l}+1\right) N_{n l} / \Gamma\left(2 A_{n l}+1\right)\right)^{2}(1 / \alpha)$ and $K_{n l}=A_{n l}+2 V_{n l}$.

\subsection{The energy shift for the MHGPs in ERQM symmetries}

The global relativistic energy shift for the improved Hellmann-generalized Morse potential model in ERQM symmetries is composed of three principal parts. The first one is produced from the effect of the generated spin-orbit effective potential. This effective potential is obtained by replacing the coupling of the angular momentum operator and the noncommutative vector $\mathbf{L} \Theta$ with the new equivalent coupling $\Theta \mathbf{L S}$ (with $\Theta^{2}=\Theta_{12}^{2}+\Theta_{23}^{2}+\Theta_{13}^{2}$ ). This degree of freedom comes considering that the infinitesimal noncommutative vector $\Theta$ is arbitrary. We have chosen it to a parallel of the spin $\mathbf{S}$ of the diatomic molecules under Hellmann-generalized Morse potential. Furthermore, we replace the new spin-orbit coupling $\Theta \mathbf{L S}$ with the corresponding physical form $(\Theta / 2) G^{2}$, with $G^{2}=J^{2}-L^{2}-S^{2}$. Moreover, in quantum mechanics, the operators ( $\widehat{H}_{r n c}^{\text {hmp }}, J^{2}$, $L^{2}, S^{2}$ and $J_{z}$ ) forms a complete set of conserved physics quantities, the eigenvalues of the operator $\mathbf{G}^{2}$ are equal to the values $\tau(j, l, s)=[j(j+1)-l(l+1)-s(s+1)] / 2$, with $|l-s| \leq j \leq|l+s|$. As a direct consequence, the partial energy shift $\Delta E_{\text {hmp }}^{s o}\left(n, \alpha, a, b, D_{e}, r_{e}, \Theta, j, l, s\right)$ due to the perturbed effective potential $V_{\text {pert }}^{\text {hmp }}(s)$ produced for the $n^{\text {th }}$ excited state, in DRKGT symmetries as follows:

$$
\Delta E_{\mathrm{hmp}}^{s o}\left(n, \alpha, a, b, D_{e}, r_{e}, \Theta, j, l, s\right)=\Theta(j(j+1)-l(l+1)-s(s+1))\langle Z\rangle_{(n l m)}^{R H M P}\left(n, n, \alpha, a, b, D_{e}, r_{e}\right) .
$$

The global expectation value $\langle Z\rangle_{(n l m)}^{R H M P}\left(n, n, \alpha, a, b, D_{e}, r_{e}\right)$ is determined from the following expression:

$$
\begin{aligned}
\langle Z\rangle_{(n l m)}^{R H M P} & \left(n, n, \alpha, a, b, D_{e}, r_{e}\right)=\left(l(l+1) \alpha^{4}\left\langle\frac{1}{(1-s)^{4}}\right\rangle_{(n l m)}-\left(E_{n l}+M\right) \alpha\left\lceil a\left\langle\frac{1}{(1-s)^{3}}\right\rangle_{(n l m)}\right.\right. \\
& -b\left\langle\frac{s}{(1-s)^{3}}\right\rangle_{(n l m)}+\left(2 D_{e} q-b\right)\left\langle\frac{s^{3 / 2}}{(1-s)^{4}}\right\rangle_{(n l m)}+2 \alpha D_{e} q(1-q)\left\langle\frac{s^{2}}{(1-s)^{3}}\right\rangle_{(n l m)} \\
& \left.\left.-2 \alpha D_{e} q^{2}\left\langle\frac{s^{3}}{(1-s)^{4}}\right\rangle_{(n l m)}\right]\right) .
\end{aligned}
$$

The second part is obtained from the magnetic effect of the perturbative effective potential $V_{\text {pert }}^{\mathrm{hmp}}(s)$ under the improved Hellmann-generalized Morse potential model. This effective potential is achieved when we replace both $\left(\mathbf{L} \Theta\right.$ and $\left.\Theta_{12}\right)$ by $\left(\sigma \aleph L_{z}\right.$ and $\left.\sigma \aleph\right)$, respectively, here ( $\aleph$ and $\sigma$ ) are symbolize the intensity of the magnetic field induced by the effect of the deformation of space-space geometry and a new infinitesimal noncommutativity parameter, so that the physical unit of the original noncommutativity parameter $\Theta_{12}$ (length) $)^{2}$ is the same unit of $\sigma \aleph$, we have also need to apply $\left\langle n^{\prime}, l^{\prime}, m^{\prime} L_{z} n, l, m\right\rangle=$ $m \delta_{m^{\prime} m} \delta_{l^{\prime} l} \delta_{n^{\prime} n}\left(-l^{\prime} \leq m^{\prime} \leq l\right.$ and $\left.-l \leq m \leq l\right)$. All of this data allows for the discovery of the new energy shift $\Delta E_{\mathrm{hmp}}^{\text {mag }}\left(n, \alpha, a, b, D_{e}, r_{e}, \sigma, m\right)$ due to the perturbed Zeeman effect which created by the influence of the improved Hellmanngeneralized Morse potential model for the $n^{t h}$ excited state in ERQM symmetries as follows:

$$
\Delta E_{\mathrm{hmp}}^{\text {mag }}\left(n, n, \alpha, a, b, D_{e}, r_{e}, \sigma, j, l, s\right)=\sigma \aleph\langle Z\rangle_{(n l m)}^{R H M P}\left(n, n, \alpha, a, b, D_{e}, r_{e}\right) m .
$$

Now, for our purposes, we are interested in finding a new third automatically important symmetry for the improved Hellmann-generalized Morse potential model at zero temperature in ERQM symmetries. This physical phenomenon is induced automatically from the influence of a perturbed effective potential $V_{\text {pert }}^{\mathrm{hmp}}(s)$ which we have seen in Eq. (33). We discover these important physical phenomena when our studied system consists of non-interacting is considered as Fermi gas, 
it is formed from all the particles in their gaseous state $\left(\mathrm{HCl}, \mathrm{LiH}, \mathrm{H}_{2}, \mathrm{ScH}, \mathrm{TiH}, \mathrm{VH}, \mathrm{CrH}, \mathrm{CuLi}, \mathrm{TiC}, \mathrm{NiC}, \mathrm{ScN}\right.$ and $\left.\mathrm{ScF}\right)$ undergoing rotation with angular velocity $\Omega$ if we make the following two simultaneous transformations to ensure that the previous calculations are not repeated:

$$
\Theta \rightarrow \chi \boldsymbol{\Omega} \quad \text { and } \quad \mathbf{L} \Theta \rightarrow \chi \mathbf{L} \boldsymbol{\Omega} .
$$

Here $\chi$ is just infinitesimal real proportional constants. We can express the effective potential $V_{\text {pert }}^{\text {hmp-rot }}(s)$ which induced the rotational movements of the diatomic molecules as follows:

$$
\begin{aligned}
V_{\text {pert }}^{\mathrm{hmp} \text {-rot }}(s) & =\left(\frac{l(l+1) \alpha^{4}}{(1-s)^{4}}-\alpha\left(E_{n l}+M\right)\left[\frac{a}{(1-s)^{3}}-\frac{b \alpha s}{(1-s)^{3}}\right.\right. \\
& \left.\left.+\left(2 D_{e} q-b\right) \frac{\alpha s}{(1-s)^{2}}+2 \frac{D_{e} \alpha q(1-q) s^{2}}{(1-s)^{3}}-2 \frac{D_{e} \alpha q^{2} s^{3}}{(1-s)^{4}}\right]\right) \chi \mathbf{L} \boldsymbol{\Omega} .
\end{aligned}
$$

To simplify the calculations without compromising physical content, we choose the rotational velocity $\Omega$ parallel to the $(O z)$ axis. Then we transform the spin-orbit coupling to the new physical phenomena as follows:

$$
\Lambda(s) \mathbf{L} \boldsymbol{\Omega}=\chi \Lambda(s) \Omega L_{z} .
$$

With

$$
\Lambda(s)=\frac{l(l+1) \alpha^{4}}{(1-s)^{4}}-\alpha\left(E_{n l}+M\right)\left(\frac{a}{(1-s)^{3}}-\frac{b \alpha s}{(1-s)^{3}}+\left(2 D_{e} q-b\right) \frac{\alpha s}{(1-s)^{2}}+2 \frac{D_{e} \alpha q(1-q) s^{2}}{(1-s)^{3}}-2 \frac{D_{e} \alpha q^{2} s^{3}}{(1-s)^{4}}\right) .
$$

All of this data allows for the discovery of the new energy shift $\Delta E_{\mathrm{hmp}}^{f-r o t}\left(n, n, \alpha, a, b, D_{e}, r_{e}, \chi, m\right)$ due to the perturbed Fermi gas effect $V_{\text {pert }}^{\mathrm{hmp}-\text { rot }}(r)$ which generated automatically by the influence of the Hellmann-generalized Morse potential for the $n^{\text {th }}$ excited state in REQM symmetries as follows:

$$
\Delta E_{\mathrm{hmp}}^{f-\operatorname{rot}}\left(n, \alpha, a, b, D_{e}, r_{e}, \chi, m\right)=\chi\langle Z\rangle_{(n l m)}^{R H M P}\left(n, \alpha, a, b, D_{e}, r_{e}, V_{2}\right) \Omega m .
$$

It is worth mentioning that the authors in Refs. [102,103] studied a rotating isotropic and anisotropic harmonically confined ultra-cold Fermi gas in a two and three-dimensional space at zero temperature, but in this study, the rotational term was added to the Hamiltonian operator, in contrast to our case, where this rotation term $\chi \Lambda(s) \mathbf{L} \boldsymbol{\Omega}$ automatically appears due to the large symmetries resulting from the deformation of space-phase.

\section{Relativistic results of IHGMP in the deformed Klien-Gordon theory symmetries}

In this section of the paper, we summarize our obtained results $\Delta E_{\mathrm{hmp}}^{s o}\left(n, \alpha, a, b, D_{e}, r_{e}, j, l, s\right), \Delta E_{\mathrm{hmp}}^{\text {mag }}\left(n, \alpha, a, b, D_{e}, r_{e}, m\right)$ and $\left.\Delta E_{\mathrm{hmp}}^{f-r o t}\left(n, \alpha, a, b, D_{e}, r_{e}, m\right)\right)$ for the $n^{t h}$ excited state due to the spin-orbital coupling, modified Zeeman effect, and perturbed Fermi gas potential induced by $V_{\text {pert }}^{\mathrm{hmp}}(s)$ on based to the superposition principle in the deformed relativistic KleinGordon theory under the improved Hellmann-generalized Morse potential model. This allows us to deduce the additive energy shift $\Delta E_{\mathrm{hmp}}^{\text {tot }}\left(n, \alpha, a, b, D_{e}, r_{e}, j, l, s, m\right)$ under the influence of the improved Hellmann-generalized Morse potential model in ERQM symmetries as follows

$$
\Delta E_{\mathrm{hmp}}^{\text {tot }}\left(n, \alpha, a, b, D_{e}, r_{e}, \Theta, \sigma, \chi, j, l, s, m\right)=\langle Z\rangle_{(n l m)}^{R H M P}\left(n, \alpha, a, b, D_{e}, r_{e}\right)(\Theta \tau(j, l, s)+\sigma \aleph m+\chi \Omega m) .
$$

The above results present the global energy shift, which generated with the effect of noncommutativity properties of spacespace; it depended explicitly on the noncommutativity parameters $(\Theta, \sigma, \chi)$, the parameters of the Hellmann-generalized Morse potential $\left(n, \alpha, a, b, D_{e}, r_{e}\right)$ in addition to the atomic quantum numbers $(j, l, s, m)$. We observed that the obtained global effective energy under the Hellmann-generalized Morse potential has a carry unit of energy because it is combined with the carrier of energy $\left(M^{2}-E_{n l}^{2}\right)$. As a direct consequence, the energy $E_{r-n c}^{\mathrm{hmp}}\left(n, n, \alpha, a, b, D_{e}, r_{e}, j, l, s, m\right)$ produced with the improved Hellmann-generalized Morse potential model, in the symmetries of ERQM is the sum of the root quart of the shift energy $\Delta\left[E_{\mathrm{hmp}}^{f-r o t}\left(n, n, \alpha, a, b, D_{e}, r_{e}, \chi, m\right)\right]^{1 / 2}$ and the relativist energy $E_{n l}$ produced by the effect due to the effect of the Hellmann-generalized Morse potential in RQM, as follows:

$$
E_{r-n c}^{\mathrm{hmp}}\left(n, n, \alpha, a, b, D_{e}, r_{e}, \Theta, \sigma, \chi, j, l, s, m\right)=E_{n l}+\left[\langle Z\rangle_{(n l m)}^{R H M P}\left(n, n, \alpha, a, b, D_{e}, r_{e}\right)(\Theta \tau(j, l, s)+\sigma \aleph m+\chi \Omega m)\right]^{1 / 2} .
$$

The relativistic energy $E_{n l}$ is determined from the energy Eq. (16.1).

Equation (47) describes the relativistic energy of some diatomic molecules such as $\mathrm{HCl}, \mathrm{LiH}, \mathrm{H}_{2}, \mathrm{ScH}, \mathrm{TiH}, \mathrm{VH}, \mathrm{CrH}$, $\mathrm{CuLi}, \mathrm{TiC}, \mathrm{NiC}, \mathrm{ScN}$ and $\mathrm{ScF}$ under the improved Hellmann-generalized Morse potential model in the DRKGT symmetries. 


\subsection{Relativistic particular cases under IHGMP in ERQM symmetries}

After examining the bound state solutions of any l-state DKGE with IHGMP, our task is now to discuss some particular cases below. By adjusting potential parameters for each case, some familiar potentials, which are useful for other physical systems, can be obtained:

1. Setting $D_{e}$ to zero the potential in Eq. (8) turns to the Hellmann potential [6-8], in RQM symmetries, as follows:

$$
V_{\mathrm{hmp}}(r) \rightarrow V_{h p}(r)=-\frac{a}{r}+\frac{b e^{-\alpha r}}{r} .
$$

The perturbed effective potential in Eq. (33) turns to perturbed effective potential in the symmetries of RNCQM as follows:

$$
V_{\text {pert }}^{h p}(s)=\left(\frac{l[l+1] \alpha^{4}}{(1-s)^{4}}-\alpha\left[E_{n l}+M\right]\left[\frac{a}{(1-s)^{3}}-\frac{b \alpha s}{(1-s)^{3}}-b \frac{\alpha s}{(1-s)^{2}}\right]\right) \mathbf{L} \Theta+O\left(\Theta^{2}\right) .
$$

In this case, the additive energy shift under the influence of the improved equally mixed Hellmann potential in ERQM symmetries is determined from the following formula:

$$
E_{\mathrm{hmp}}^{f-r o t}\left(n, n, \alpha, a, b, D_{e}, r_{e}, \chi, m\right)=\langle Z\rangle_{(n l m)}^{R H P}(n, n, \alpha, a, b)(\Theta \tau(j, l, s)+\sigma \aleph m+\chi \Omega m) .
$$

Thus, the corresponding global expectation value $\langle Z\rangle_{(n l m)}^{R H P}(n, n, \alpha, a, b)$ is determined from the following expression:

$$
\begin{aligned}
\langle Z\rangle_{\substack{(n l m) \\
R H M P}}(n, n, \alpha, a, b) & =\left(l(l+1) \alpha^{4}\left\langle\frac{1}{(1-s)^{4}}\right\rangle_{(n l m)}\right. \\
& \left.-\left(E_{n l}+M\right) \alpha\left[a\left\langle\frac{1}{(1-s)^{3}}\right\rangle_{(n l m)}-b\left\langle\frac{s}{(1-s)^{3}}\right\rangle_{(n l m)}-b\left\langle\frac{s^{3 / 2}}{(1-s)^{4}}\right\rangle_{(n l m)}\right]\right) .
\end{aligned}
$$

The new relativistic energy in Eq. (47) reduces to the new energy $E_{r-n c}^{h p}(n, n, \alpha, a, b, \Theta, \sigma, \chi, j, l, s, m)$ under modified equal scalar and vector improved Hellmann potential in ERQM symmetries, as follows:

$$
E_{r-n c}^{h p}(n, n, \alpha, a, b, \Theta, \sigma, \chi, j, l, s, m)=E_{n l}^{h p}+\left[\langle Z\rangle_{(n l m)}^{R H P}(n, n, \alpha, a, b)(\Theta \tau(j, l, s)+\sigma \aleph m+\chi \Omega m)\right]^{1 / 2} .
$$

Making the corresponding parameter replacements in Eq. (16.1), we obtain the energy equation for the improved Hellmann potential in the Klein-Gordon theory with equally mixed potentials in RQM symmetries as:

$$
\begin{aligned}
E_{n l}^{h p 2}-M^{2} & =\left(D_{e}-\alpha a\right)\left(E_{n l}^{h p}+M\right)+\alpha^{2} l(l+1) \\
& -\frac{1}{4}\left(\frac{\alpha\left(n+\frac{1}{2}+\delta_{n l}\right)-\left(E_{n l}^{h p}+M\right)(a-b)}{n+\frac{1}{2}+l(l+1)}-\frac{\alpha l(l+1)\left(E_{n l}^{h p}+M\right)}{n+\frac{1}{2}+l(l+1)}\right)^{2} .
\end{aligned}
$$

2. Setting the parameters $a=b=0$, the potential in Eq. (8) turns to the equal scalar and vector generalized Morse potential or Deng-Fan potential [21], in RQM symmetries, as follows:

$$
V_{m p}(r)=D_{e}-2 \frac{D_{e} q e^{-\alpha r}}{1-e^{-\alpha r}}+\frac{D_{e} q^{2} e^{-2 \alpha r}}{\left(1-e^{-\alpha r}\right)^{2}} .
$$

The perturbed effective potential in Eq. (33) turns to perturbed effective potential in the symmetries of RNCQM as follows: 
$V_{\text {pert }}^{m p}(s)=\left(\frac{l[l+1] \alpha^{4}}{[1-s]^{4}}-\alpha\left[E_{n l}+M\right]\left[2 D_{e} q \frac{\alpha s}{(1-s)^{2}}+2 \frac{D_{e} \alpha q(1-q) s^{2}}{(1-s)^{3}}-2 \frac{D_{e} \alpha q^{2} s^{3}}{(1-s)^{4}}\right]\right) \mathbf{L} \Theta+O\left(\Theta^{2}\right)$.

In this case, the additive energy shift under the influence of improved equally mixed generalized Morse potentials in REQM symmetries is given by:

$$
\Delta E_{m p}^{t o t}\left(n, \alpha, D_{e}, r_{e}, \Theta, \sigma, \chi, j, l, s, m\right)=\langle Z\rangle_{(n l m)}^{R M P}\left(n, \alpha, D_{e}, r_{e}\right)(\Theta \tau(j, l, s)+\sigma \aleph m+\chi \Omega m) .
$$

Thus, the corresponding global expectation value is determined from the following expression:

$$
\begin{aligned}
\langle Z\rangle_{(n l m)}^{R M P}\left(n, \alpha, a, b, D_{e}, r_{e}\right) & =\left(l(l+1) \alpha^{4}\left\langle\frac{1}{(1-s)^{4}}\right\rangle_{(n l m)}-2 D_{e} q\left(E_{n l}+M\right) \alpha\right. \\
& \left.\times\left[\left\langle\frac{s^{3 / 2}}{(1-s)^{4}}\right\rangle_{(n l m)}+\alpha(1-q)\left\langle\frac{s^{2}}{(1-s)^{3}}\right\rangle_{(n l m)}-\alpha q\left\langle\frac{s^{3}}{(1-s)^{4}}\right\rangle_{(n l m)}\right]\right) .
\end{aligned}
$$

The new relativistic energy in Eq. (47) reduces to the new energy under improved equal scalar and vector Hellmann potentials in RNCQM, as follows:

$$
E_{r-n c}^{\mathrm{hmp}}\left(n, \alpha, D_{e}, r_{e}, \Theta, \sigma, \chi, j, l, s, m\right)=E_{n l}^{m p}+\left[\langle Z\rangle_{(n l m)}^{R M P}\left(n, n, \alpha, D_{e}, r_{e}\right)(\Theta \tau(j, l, s)+\sigma \aleph m+\chi \Omega m)\right]^{1 / 2} .
$$

Making the corresponding parameter replacements in Eq. (16.1), we obtain the energy equation for the generalized Morse potential in the Klein-Gordon theory in RQM symmetries as:

$$
\begin{aligned}
E_{n l}^{m p 2}-M^{2} & =D_{e}\left(E_{n l}+M\right)+\alpha^{2} l(l+1)-\frac{1}{4}\left(\frac{\alpha\left(n+\frac{1}{2}+\delta_{n l}\right)-2 \frac{D_{e}}{\alpha}\left(e^{\alpha r_{e}}-1\right)\left(E_{n l}^{m p}+M\right)}{n+\frac{1}{2}+l(l+1)}\right. \\
& \left.-\frac{\left(E_{n l}^{m p}+M\right)\left(\frac{D_{e}}{\alpha}\left(e^{\alpha r_{e}}-1\right)^{2}+\alpha l(l+1)\right)}{n+\frac{1}{2}+l(l+1)}\right)^{2} \cdot
\end{aligned}
$$

\section{Fermionic massive spin $1 / 2$ particles interacting with relativistic IHGMP model in the deformed Dirac theory}

To obtain the improved Dirac equation (IDE) for the improved Hellmann-generalized Morse potential model in the symmetries of deformed Dirac theory (DDT), we replace both the ordinary Hamiltonian operators $\widehat{H}\left(p_{i}, x_{i}\right)$, ordinary spinors $\Psi_{n k}(r, \theta, \varphi)$, and ordinary energy $E_{n k}$ by NC Hamiltonian operator $\widehat{H}\left(p_{n c i}, x_{n c i}\right)$, the new spinor $\Psi_{n k}\left(\vec{r}_{n c}\right)$, and new energy $E_{n c-n k}^{m p}$ and the ordinary product will be replaced by star product $*$, respectively. This allows us to write the IED for improved Hellmann-generalized Morse potential as follows [88-91]:

$$
\widehat{H}\left(p_{n c i}, x_{n c i}\right) * \Psi_{n k}\left(\vec{r}_{n c}\right)=E_{n c-n k}^{m p} \Psi_{n k}\left(\vec{r}_{n c}\right) .
$$

It is worth motioning that Bopp's shift method permutes to reduce the above equation to the simplest form:

$$
H_{m p}\left(p_{n c i}, x_{n c i}\right) \Psi_{n k}\left(\vec{r}_{n c}\right)=E_{n c-n k}^{m p} \Psi_{n k}(\vec{r}) .
$$

The new Hamiltonian operator for fermionic massive spin $1 / 2$ particles interacting with the relativistic improved Hellmanngeneralized Morse potential model is given by:

$$
H_{m p}\left(p_{n c i}, x_{n c i}\right)=\alpha p_{n c}+\beta\left(M+S_{\mathrm{hmp}}\left(r_{n c}\right)\right)+V_{\mathrm{hmp}}\left(r_{n c}\right) .
$$


By applying the notion of the Weyl-Moyal star product which has been seen previously in Eqs. (2)-(4), the differential equations that are satisfied by the radial wave function in Eqs. (13.2) and (13.3), for the spin symmetry solutions and pseudospin symmetry of MDE with IHGMP, respectively, in relativistic noncommutative quantum mechanics symmetries in the deformed Dirac theory (RNCQM-DT, in short), becomes as follows:

$$
\begin{gathered}
{\left[\frac{d^{2}}{d r^{2}}-\frac{k(k+1)}{r_{n c}^{2}}-\left(M+E_{n k}^{s}-C_{s}\right)\left(M-E_{n k}^{s}+\Sigma_{\mathrm{hmp}}\left(r_{n c}\right)\right)\right] F_{n k}(r)=0,} \\
{\left[\frac{d^{2}}{d r^{2}}-\frac{k(k-1)}{r_{n c}^{2}}-\left(M+E_{n k}^{p s}-\Delta_{\mathrm{hmp}}\left(r_{n c}\right)\right)\left(M-E_{n k}^{p s}+C_{p s}\right)\right] G_{n k}(r)=0,}
\end{gathered}
$$

with

$$
\Sigma_{\mathrm{hmp}}\left(r_{n c}\right)=\Delta_{\mathrm{hmp}}\left(r_{n c}\right)=D_{e}-\frac{a}{r_{n c}}+\frac{b e^{-\alpha r_{n c}}}{r_{n c}}+D_{e}\left(1+\frac{q}{e^{-\alpha r_{n c}}-1}\right)^{2} .
$$

The new operators $\Sigma_{\mathrm{hmp}}\left(r_{n c}\right), \Delta_{\mathrm{hmp}}\left(r_{n c}\right),\left(k(k+1) / r_{n c}^{2}\right)$ and $\left(k(k-1) / r_{n c}^{2}\right)$ in the deformed Dirac theory symmetries, are expressed as:

$$
\begin{aligned}
\Sigma_{\mathrm{hmp}}\left(r_{n c}\right) & =D_{e}-\frac{a}{r}+\frac{b e^{-\alpha r}}{r}+D_{e}\left(1+\frac{q}{e^{-\alpha r}-1}\right)^{2}-\frac{\partial \Sigma_{\mathrm{hmp}}(r)}{\partial r} \frac{\mathbf{L} \Theta}{2 r}+O\left(\Theta^{2}\right), \\
\frac{k(k-1)}{r_{n c}^{2}} & =\frac{k(k-1)}{r^{2}}+\frac{k(k-1)}{r^{4}} \mathbf{L} \Theta+O\left(\Theta^{2}\right),
\end{aligned}
$$

and

$$
\frac{k(k+1)}{r_{n c}^{2}}=\frac{k(k+1)}{r^{2}}+\frac{k(k+1)}{r^{4}} \mathbf{L} \Theta+O\left(\Theta^{2}\right) .
$$

Substituting Eqs. (65) and (66) into Eqs. (63.1) and (63.2), we obtain:

$$
\begin{aligned}
\left(\frac{d^{2}}{d r^{2}}-\frac{k(k+1)}{r^{2}}-\right. & \left(M+E_{n k}^{s p}-C_{s}\right)\left(M-E_{n k}^{s p}+\Sigma_{\mathrm{hmp}}(r)\right) \\
- & {\left.\left[\frac{k(k+1)}{r^{4}}-\frac{\left(M+E_{n k}^{s p}-C_{s}\right)}{2 r} \frac{\partial \Sigma_{\mathrm{hmp}}(r)}{\partial r}\right] \mathbf{L} \Theta\right) F_{n k}(r)=0, } \\
\left(\frac{d^{2}}{d r^{2}}-\frac{k(k-1)}{r^{2}}-\right. & \left(M+E_{n k}^{p s}-\Delta_{\mathrm{hmp}}(r)\right)\left(M-E_{n k}^{p s}+C_{p s}\right) \\
& \left.-\left[\frac{k(k-1)}{r^{4}}-\frac{\left(M-E_{n k}^{p s}+C_{p s}\right)}{2 r} \frac{\partial \Delta_{\mathrm{hmp}}(r)}{\partial r}\right] \mathbf{L} \Theta\right) G_{n \widetilde{k}}(r)=0 .
\end{aligned}
$$

By comparing (Eqs. (63.1) and (63.2)) and (Eqs. (69.1) and (69.2)), we get an effective deformed spin symmetry $\Sigma_{\text {hmp }}(r)$ and pseudospin symmetry $\Delta_{\text {hmp }}(r)$, similar to the perturbative effective potential of Eq. (28),

$$
\begin{aligned}
& \Sigma_{\mathrm{hmp}}^{\text {pert }}(r)=\frac{k(k+1)}{r^{4}}-\frac{\left(M+E_{n k}^{s p}-C_{s}\right)}{2 r} \frac{\partial \Sigma_{\mathrm{hmp}}(r)}{\partial r} \mathbf{L} \Theta, \\
& \Delta_{\mathrm{hmp}}^{\text {pert }}(r)=\frac{k(k+1)}{r^{4}}-\frac{\left(M-E_{n k}^{s p}+C_{p s}\right)}{2 r} \frac{\partial \Delta_{\mathrm{hmp}}(r)}{\partial r} \mathbf{L} \Theta .
\end{aligned}
$$

A direct calculation gives:

$$
\frac{\partial \Sigma_{\mathrm{hmp}}(r)}{\partial r}=\frac{a}{r^{2}}+\frac{b \alpha e^{-\alpha r}}{r}-\frac{b e^{-\alpha r}}{r^{2}}+2 D_{e} \alpha q \frac{e^{-\alpha r}}{\left(1-e^{-\alpha r}\right)^{2}}-2 D_{e} \alpha q^{2} \frac{e^{-\alpha r}}{\left(1-e^{-\alpha r}\right)^{3}} .
$$


We apply the Greene and Aldrich approximation to the Eqs. (70.1) and (70.2) to obtain:

$$
\begin{aligned}
\Sigma_{\mathrm{hmp}}^{\text {pert }}(r) & =\alpha^{2}\left(\frac{k[k+1] \alpha^{2}}{[1-s]^{4}}-\left[M+E_{n k}^{s p}-C_{s}\right]\right. \\
& \left.\times\left[\frac{a \alpha}{2\{1-s\}^{3}}-\frac{b \alpha s}{2\{1-s\}^{2}}-\frac{\left\{D_{e} q-b \alpha / 2\right\} s}{\{1-s\}^{3}}-D q^{2} \frac{s}{\{1-s\}^{4}}\right]\right) \mathbf{L} \Theta, \\
\Delta_{\mathrm{hmp}}^{\text {pert }}(r) & =\alpha^{2}\left(\frac{k[k+1] \alpha^{2}}{[1-s]^{4}}-\left[M-E_{n k}^{p s}-C_{p s}\right]\right. \\
& \left.\times\left[\frac{a \alpha}{2\{1-s\}^{3}}-\frac{b \alpha s}{2\{1-s\}^{2}}-\frac{\left\{D_{e} q-b \alpha / 2\right\} s}{\{1-s\}^{3}}-D q^{2} \frac{s}{\{1-s\}^{4}}\right]\right) \mathbf{L} \Theta .
\end{aligned}
$$

Thus, we need to find the expectation values of the radial terms $\left(1 /[1-s]^{4}\right),\left(1 /[1-s]^{3}\right),\left(s /[1-s]^{2}\right),\left(s /[1-s]^{3}\right)$ and $\left(s /[1-s]^{4}\right)$ taking into account the wave function which we have seen previously in Eqs. (15.2) and (15.3). We have calculated the expectation values of the radial terms of the first four terms, and but for the last term, we have not done so yet because it did not appear in the Klein Gordon deformed theory using the wave function in Eq. (15.1). To avoid repeating the previous work, it is sufficient to make the following changes to find the four first expectation values as follows:

$$
\begin{aligned}
& F_{n k}(r)=\frac{N_{n k}\left(2 \omega_{n k}+1\right)_{n}}{n !} s^{\omega_{n k}}(1-s)^{\lambda_{n k}+\frac{1}{2}}{ }_{2} F_{1}\left(-n, n+2 \omega_{n k}+2 \lambda_{n k}+1 ; 1+\omega_{n k} ; s\right) Y_{l}^{m}(\Omega) \\
& G_{n \tilde{k}}(r)=\frac{N_{n k}\left(2 \Omega_{n k}+1\right)_{n}}{n !} s^{\Omega_{n k}}(1-s)^{\beta_{n l}+\frac{1}{2}}{ }_{2} F_{1}\left(-n, n+2 \Omega_{n k}+2 \beta_{n l}+1 ; 1+\Omega_{n k} ; s\right) Y_{l}^{m}(\Omega) \\
& \left\{A_{n l} / 2 \rightarrow \omega_{n k}\left(\Omega_{n k}\right)\right. \text { and } \\
& V_{n l} \rightarrow \lambda_{n k}\left(\beta_{n k}\right) \frac{n ! \Gamma\left(n+A_{n l}+1\right) N_{n l}}{\Gamma\left(2 A_{n l}+1\right)} \rightarrow \frac{N_{n k}\left(2 \omega_{n k}+1\right)_{n}}{n !}\left(\frac{N_{n k}\left(2 \Omega_{n k}+1\right)_{n}}{n !}\right) .
\end{aligned}
$$

Then the recorded results in relations $(37 . \mathrm{i}=1,2,3,4)$ will turn out to be appropriate to Dirac's deformed theory:

$$
\begin{aligned}
& \left\langle\frac{1}{(1-s)^{4}}\right\rangle_{(n l m)}^{s p}=N_{n k}^{v i r} \frac{\Gamma\left(2 \omega_{n k}\right) \Gamma\left(2 \lambda_{n k}-2\right)}{\Gamma\left(K_{n k}^{n e w}-2\right)} \\
& \times{ }_{3} F_{2}\left(-n, n+2 \omega_{n k}+\lambda_{n k}+1,2 \lambda_{n k}-2 ; 1+2 \omega_{n k}, K_{n k}^{n e w}-2 ; 1\right), \\
& \left\langle\frac{1}{(1-s)^{3}}\right\rangle_{(n l m)}^{s p}=N_{n k}^{v i r} \frac{\Gamma\left(2 \omega_{n k}\right) \Gamma\left(2 \lambda_{n k}-1\right)}{\Gamma\left(K_{n k}^{n e w}-1\right)} \\
& \times{ }_{3} F_{2}\left(-n, n+2 \omega_{n k}+\lambda_{n k}+1,2 \lambda_{n k}-1 ; 1+2 \omega_{n k}, K_{n k}^{n e w}-1 ; 1\right), \\
& \left\langle\frac{s}{(1-s)^{3}}\right\rangle_{(n l m)}^{s p}=N_{n k}^{v i r} \frac{\Gamma\left(2 \omega_{n k}+1\right) \Gamma\left(2 \lambda_{n k}-1\right)}{\Gamma\left(K_{n k}^{n e w}\right)} \\
& \times{ }_{3} F_{2}\left(-n, n+2 \omega_{n k}+\lambda_{n k}+1,2 \lambda_{n k}-1 ; 1+2 \omega_{n k}, K_{n k}^{n e w} ; 1\right), \\
& \left\langle\frac{s}{(1-s)^{2}}\right\rangle_{(n l m)}^{s p}=N_{n k}^{v i r} \frac{\Gamma\left(2 \omega_{n k}+1\right) \Gamma\left(2 \lambda_{n k}\right)}{\Gamma\left(K_{n k}^{n e w}+1\right)} \\
& \times{ }_{3} F_{2}\left(-n, n+2 \omega_{n k}+\lambda_{n k}+1,2 \lambda_{n k}-1 ; 1+2 \omega_{n k}, K_{n k}^{n e w}+1 ; 1\right), \\
& \left\langle\frac{1}{(1-s)^{4}}\right\rangle_{(n l m)}^{p s p}=N_{n k}^{\prime v i r} \frac{\Gamma\left(2 \Omega_{n k}\right) \Gamma\left(2 \beta_{n k}-2\right)}{\Gamma\left(K_{n k}^{\prime n e w}-2\right)} \\
& \times{ }_{3} F_{2}\left(-n, n+2 \Omega_{n k}+\beta_{n k}+1,2 \beta_{n k}-2 ; 1+2 \Omega_{n k}, K_{n k}^{\text {new }}-2 ; 1\right),
\end{aligned}
$$




$$
\begin{aligned}
\left\langle\frac{1}{(1-s)^{3}}\right\rangle_{(n l m)}^{p s p} & =N_{n k}^{\prime v i r} \frac{\Gamma\left(2 \Omega_{n k}\right) \Gamma\left(2 \beta_{n k}-1\right)}{\Gamma\left(K_{n k}^{\prime n e w}-1\right)} \\
& \times{ }_{3} F_{2}\left(-n, n+2 \Omega_{n k}+\beta_{n k}+1,2 \beta_{n k}-1 ; 1+2 \Omega_{n k}, K_{n k}^{\prime n e w}-1 ; 1\right), \\
\left\langle\frac{s}{(1-s)^{3}}\right\rangle_{(n l m)}^{p s p} & =N_{n k}^{\prime v i r} \frac{\Gamma\left(2 \Omega_{n k}+1\right) \Gamma\left(2 \beta_{n k}-1\right)}{\Gamma\left(K_{n k}^{\prime n e w}\right)} \\
& \times{ }_{3} F_{2}\left(-n, n+2 \Omega_{n k}+\beta_{n k}+1,2 \Omega_{n k}-1 ; 1+2 \Omega_{n k}, K_{n k}^{\prime n e w} ; 1\right), \\
\left\langle\frac{s}{(1-s)^{2}}\right\rangle_{(n l m)}^{p s p} & =N_{n k}^{\prime v i r} \frac{\Gamma\left(2 \Omega_{n k}+1\right) \Gamma\left(2 \beta_{n k}\right)}{\Gamma\left(K_{n k}^{\prime n e w}+1\right)} \\
& \times{ }_{3} F_{2}\left(-n, n+2 \Omega_{n k}+\beta_{n k}+1,2 \beta_{n k}-1 ; 1+2 \Omega_{n k}, K_{n k}^{\text {nnew }}+1 ; 1\right),
\end{aligned}
$$

with

$$
N_{n k}^{v i r}=\left(\frac{n ! \Gamma\left(n+2 \omega_{n k}+1\right) N_{n k}}{\Gamma\left(4 \omega_{n k}+1\right)}\right)^{2} \frac{1}{\alpha}, \quad N_{n k}^{v i r}=\left(\frac{n ! \Gamma\left(n+2 \Omega_{n k}+1\right) N_{n k}}{\Gamma\left(4 \Omega_{n k}+1\right)}\right)^{2} \frac{1}{\alpha},
$$

$K_{n k}^{n e w}=2 \omega_{n k}+2 \lambda_{n k}$ and $K_{n k}^{\prime n e w}=2 \Omega_{n k}+2 \beta_{n k}$. The four first results (74.1), (74.2), (74.3) and (74.4) are present the expectation values for deformed spin symmetry while the last four terms (74.5), (74.6), (74.7) and (74.8) related to the pseudospin symmetry. Now, the expectation value for $\left(s /[1-s]^{4}\right)$ deformed spin symmetry and pseudospin symmetry is determined from the equation taking into account the wave Eqs. (15.2) and (15.3) as follows:

$$
\begin{aligned}
\left\langle\frac{s}{(1-s)^{4}}\right\rangle_{(n l m)}^{s p} & =N_{n k}^{v i r} \int_{0}^{+1} s^{2 \omega_{n k}}(1-s)^{2 \lambda_{n k}-3}\left[{ }_{2} F_{1}\left(-n, n+2 \omega_{n k}+2 \lambda_{n k}+1 ; 1+\omega_{n k} ; s\right)\right]^{2} d s, \\
\left\langle\frac{s}{(1-s)^{4}}\right\rangle_{(n l m)}^{p s p} & =N_{n k}^{v i r} \int_{0}^{+1} s^{2 \Omega_{n k}}(1-s)^{2 \beta_{n k}-3}\left[{ }_{2} F_{1}\left(-n, n+2 \Omega_{n k}+2 \beta_{n l}+1 ; 1+\Omega_{n k} ; s\right)\right]^{2} d s .
\end{aligned}
$$

With the help of the special integral that we saw in Eq. (36), we obtain easily:

$$
\begin{aligned}
& \left\langle\frac{s}{(1-s)^{4}}\right\rangle_{(n l m)}^{s p}=N_{n k}^{v i r} \frac{\Gamma\left(2 \omega_{n k}+1\right) \Gamma\left(2 \lambda_{n k}-2\right)}{\Gamma\left(K_{n k}^{n e w}-1\right)}{ }_{3} F_{2}\left(-n, n+K_{n k}^{n e w}+1,2 \lambda_{n k}-2 ; 1+\omega_{n k}, K_{n k}^{n e w}-1 ; 1\right), \\
& \left\langle\frac{s}{(1-s)^{4}}\right\rangle_{(n l m)}^{p s p}=N_{n k}^{\prime v i r} \frac{\Gamma\left(2 \Omega_{n k}+1\right) \Gamma\left(2 \beta_{n k}-2\right)}{\Gamma\left(K_{n k}^{\prime n e w}-1\right)}{ }_{3} F_{2}\left(-n, n+K_{n k}^{\text {new }}+1,2 \beta_{n k}-2 ; 1+\Omega_{n k}, K_{n k}^{\text {new }}-1 ; 1\right) .
\end{aligned}
$$

Moreover, and by applying the same method that we saw in the previous section related to the deformed Klein-Gordon theory, taking into account statistical differences between this theory and deformed Dirac theory. The global additive energy $\Delta E_{\mathrm{hmp}}^{D-\text { tot }}\left(n, \alpha, a, b, D_{e}, r_{e}, \Theta, \sigma, \chi, j, l, s, m\right)$, which produced with effective two perturbative terms $\Sigma_{\text {hmp }}^{\text {pert }}(r)$ and $\Delta_{\text {hmp }}^{\text {pert }}(r)$ for deformed spin symmetry and pseudospin symmetry, in RNCQM-DT symmetries, is as follows

$$
\begin{aligned}
\Delta E_{\mathrm{hmp}}^{t o t}\left(n, \alpha, a, b, D_{e}, r_{e}, \Theta, \sigma, \chi, j, l, s, m\right) & =(\Theta \tau(j, l, s)+\sigma \aleph m+\chi \Omega m) \\
& \times\left\{\begin{array}{l}
\langle Z\rangle_{(n l m)}^{s p}\left(n, n, \alpha, a, b, D_{e}, r_{e}\right) \quad \text { For deformed spin symmetry } \\
\langle Z\rangle_{(n l m)}^{p s}\left(n, n, \alpha, a, b, D_{e}, r_{e}\right) \text { For deformed p-spin symmetry }
\end{array},\right.
\end{aligned}
$$

where $\langle Z\rangle_{(n l m)}^{s p}\left(n, n, \alpha, a, b, D_{e}, r_{e}\right)$ and $\langle Z\rangle_{(n l m)}^{p s}\left(n, n, \alpha, a, b, D_{e}, r_{e}\right)$ are determined by the following relations

$$
\begin{gathered}
\langle Z\rangle_{(n l m)}^{s p}\left(n, n, \alpha, a, b, D_{e}, r_{e}\right)=\alpha^{2}\left(k(k+1) \alpha^{2}\left\langle\frac{1}{(1-s)^{4}}\right\rangle_{(n l m)}^{s p}-\left[M+E_{n k}^{s p}-C_{s}\right]\left[\frac{a \alpha}{2}\left\langle\frac{1}{(1-s)^{3}}\right\rangle_{(n l m)}^{s p}\right.\right. \\
\left.\left.-\frac{b \alpha s}{2}\left\langle\frac{s}{(1-s)^{2}}\right\rangle_{(n l m)}^{s p}-\left(D_{e} q-b \alpha / 2\right)\left\langle\frac{s}{(1-s)^{3}}\right\rangle_{(n l m)}^{s p}-D q^{2}\left\langle\frac{s}{(1-s)^{4}}\right\rangle_{(n l m)}^{s p}\right]\right) .
\end{gathered}
$$


and

$$
\begin{aligned}
\langle Z\rangle_{(n l m)}^{p s} & \left(n, n, \alpha, a, b, D_{e}, r_{e}\right)=\alpha^{2}\left(k(k-1) \alpha^{2}\left\langle\frac{1}{(1-s)^{4}}\right\rangle_{(n l m)}^{s p}-\left(M-E_{n k}^{p s}-C_{p s}\right)\left[\frac{a \alpha}{2}\left\langle\frac{1}{(1-s)^{3}}\right\rangle_{(n l m)}^{p s}\right.\right. \\
& \left.\left.-\frac{b \alpha s}{2}\left\langle\frac{s}{(1-s)^{2}}\right\rangle_{(n l m)}^{p s}-\left(D_{e} q-b \alpha / 2\right)\left\langle\frac{s}{(1-s)^{3}}\right\rangle_{(n l m)}^{p s}-D q^{2}\left\langle\frac{s}{(1-s)^{4}}\right\rangle_{(n l m)}^{p s}\right]\right) .
\end{aligned}
$$

We have seen that in the previous section that the eigenvalues of the operator $\mathbf{G}^{2}=\mathbf{J}^{2}-\mathbf{L}^{2}-\mathbf{S}^{2}$ are equal to the values $\tau(j, l, s)=[j(j+1)-l(l+1)-3 / 4] / 2$ and $\tau(j, \widetilde{l}, \widetilde{s})=[j(j+1)-\widetilde{l}(l+1)-3 / 4] / 2$, thus, for the case of spin- $1 / 2$, the possible values of $j$ are $l \pm 1 / 2$ and $\widetilde{l} \pm 1 / 2$ for spin symmetry $\tau(j, l, s)$ and pseudospin symmetry $\tau(j, \widetilde{l}, \widetilde{s})$, which allows us to get and as follows:

$$
\tau(j=l \pm 1 / 2, l, s=1 / 2)=\left\{\begin{array}{rl}
\frac{l}{2} & \text { For up polarity } j=l+1 / 2 \\
-\frac{l+1}{2} & \text { For dawn polarity } j=l-1 / 2
\end{array},\right.
$$

and

$$
\tau(j=\widetilde{l} \pm 1 / 2, \widetilde{l}, \widetilde{s}=1 / 2)=\left\{\begin{array}{ccc}
\frac{\tilde{l}}{2} & \text { For up polarity } \quad j=\widetilde{l}+1 / 2 \\
-\frac{\tilde{l}+1}{2} & \text { For dawn polarity } \quad j=\widetilde{l}-1 / 2
\end{array} .\right.
$$

The new relativistic energy $E_{r-n c}^{s p}\left(n, \alpha, a, b, D_{e}, r_{e}, \Theta, \sigma, \chi, j, l, s, m\right)$ and $E_{r-n c}^{p s}\left(n, \alpha, a, b, D_{e}, r_{e}, \Theta, \sigma, \chi, j, \widetilde{l}, \widetilde{s}, m\right)$ for the case of spin-1/2 with improved Hellmann-generalized Morse potential, in the symmetries of the deformed Dirac symmetries, corresponding to the generalized $n^{\text {th }}$ excited states:

$$
\begin{aligned}
& \left.E_{r-n c}^{s p}\left(n, \alpha, a, b, D_{e}, r_{e}, \Theta, \sigma, \chi, j, l, s, m\right)=E_{n k}^{s}+\langle Z\rangle_{(n l m)}^{s p} n, n, \alpha, a, b, D_{e}, r_{e}\right) \\
& \times\left\{\begin{array}{rl}
\frac{l}{2} & \text { For up polarity } j=l+1 / 2 \\
-\frac{l+1}{2} & \text { For dawn polarity } j=l-1 / 2
\end{array},\right.
\end{aligned}
$$

and

$$
\begin{aligned}
& E_{r-n c}^{p s}\left(n, \alpha, a, b, D_{e}, r_{e}, \Theta, \sigma, \chi, j, \widetilde{l}, \widetilde{s}, m\right)=E_{n k}^{p s}+\langle Z\rangle_{(n l m)}^{p s}\left(n, n, \alpha, a, b, D_{e}, r_{e}\right) \\
& \times\left\{\begin{aligned}
\frac{\widetilde{l}}{2} & \text { For up polarity } \quad j=\widetilde{l}+1 / 2 \\
-\frac{\vec{l}+1}{2} & \text { For dawn polarity } j=\widetilde{l}-1 / 2
\end{aligned}\right.
\end{aligned}
$$

where $E_{n k}^{s}$ and $E_{n k}^{p s}$ are usual relativistic energies within the Dirac theory obtained from Eqs. (16.3) and (16.4) while $k$ and $\widetilde{k}$ are determined from the following relations:

$$
k=\left\{\begin{array}{lll}
k_{1}=-(l+1)=l+1 / 2 \quad \text { For } & s_{1 / 2}, p_{3 / 2} \ldots \text { etc. } \\
j=l+1 / 2 \quad \text { Aligned spin } & k<0 \\
k_{1}=-(l+1)=l+1 / 2 & \text { For } & s_{1 / 2}, p_{3 / 2} \ldots \text { etc. } \\
j=l-1 / 2 \quad & \text { Aligned spin } & k>0
\end{array},\right.
$$


and

$$
\widetilde{k}=\left\{\begin{array}{l}
k_{1}=-\widetilde{l}=-(j+1 / 2) \quad \text { For } \quad s_{1 / 2}, p_{3 / 2} \ldots \text { etc. } \\
j=\widetilde{l}-1 / 2 \quad \text { Aligned spin } \widetilde{k}<0 \\
k_{1}=-(l+1)=l+1 / 2 \quad \text { For } \quad s_{1 / 2}, p_{3 / 2} \ldots \text { etc. } \\
j=l-1 / 2 \quad \text { Un aligned spin } \widetilde{k}>0
\end{array} .\right.
$$

\section{Nonrelativistic study of improved Hellmann-generalized Morse potential}

\subsection{Nonrelativistic improved Hellmann-generalized Morse potential}

In this subsection section, we want to derive the nonrelativistic spectrum, which is produced by the effect of the IHGMP for some diatomic molecules such as $\mathrm{HCl}, \mathrm{LiH}, \mathrm{H} 2, \mathrm{ScH}, \mathrm{TiH}, \mathrm{VH}, \mathrm{CrH}, \mathrm{CuLi}, \mathrm{TiC}, \mathrm{NiC}, \mathrm{ScN}$, and $\mathrm{ScF}$ by applying the notion of the Weyl Moyal star product which have seen previously in Eqs. (2.1), (2.2), and (4) to the differential equation that satisfied by the radial wave function $\psi_{n l}(r)$ in Eq. (13.4), the radial wave function in extended nonrelativistic quantum mechanics (ENRQM, for short) symmetries becomes as follows:

$$
\left(\frac{d^{2}}{d r^{2}}+2 M\left[E_{n l}^{n r}-V_{\mathrm{hmp}}\{r\}-\frac{l\{l+1\}}{2 M r^{2}}\right]\right) * \psi_{n l}(r)=0 .
$$

According to Bopp's shift method, Eq. (84) becomes similar to the following like the Schrödinger equation (without the notion of the Weyl-Moyal star product):

$$
\left(\frac{d^{2}}{d r^{2}}+2 M\left[E_{n l}^{n r}-V_{\mathrm{hmp}}\left\{r_{n c}\right\}-\frac{l\{l+1\}}{2 M r_{n c}^{2}}\right]\right) \psi_{n l}(r)=0 .
$$

From Eqs. (1.2) and (23) we can write this potential in the ENRQM symmetries as follows:

$$
V_{\mathrm{hmp}}\left(r_{n c}\right)=D_{e}-\frac{a}{r}+\frac{b e^{-\alpha r}}{r}-\frac{b e^{-\alpha r}}{r^{2}}+2 D_{e} q \frac{e^{-\alpha r}}{\left(1-e^{-\alpha r}\right)^{2}}+D_{e} q^{2} \frac{e^{-\alpha r}}{\left(1-e^{-\alpha r}\right)^{2}}+V_{n r-p e r t}^{\mathrm{hmp}}(r),
$$

where $V_{n r-p e r t}^{\mathrm{hmp}}(r)$ is infinitesimal value if compared with the main part $V_{\mathrm{hmp}}(r)$. Furthermore, it presents the global perturbative potential of IHGMP in the ENRQM symmetries:

$$
V_{n r-p e r t}^{\mathrm{hmp}}(r)=\frac{l(l+1)}{r^{4}} \mathbf{L} \Theta-\frac{\partial V_{\mathrm{hmp}}(r)}{\partial r} \frac{\mathbf{L} \Theta}{2 r}+O\left(\Theta^{2}\right) .
$$

The first term in Eq. (87) due to the centrifugal term $\left(l[l+1] / r_{n c}^{2}\right)$ in ENRQM symmetries (see Eq. (24)) which equals the usual centrifugal term $\left(l[l+1] / r^{2}\right)$ plus the perturbative centrifugal term $\left(l[l+1] / r^{4}\right) \mathbf{L} \Theta$ while the second term in Eq. (87) is produced with the effect of IHGMP. We have seen in Eq. (31) the expression $\partial V_{\mathrm{hmp}}(r) / \partial r$, allows us to get as follows:

$$
\begin{aligned}
V_{n r-p e r t}^{\mathrm{hmp}}(r) & =\frac{l(l+1)}{r^{4}} \mathbf{L} \Theta-\left(\frac{a}{r^{2}}-\frac{b \alpha e^{-\alpha r}}{r}-\frac{b \alpha e^{-\alpha r}}{r^{2}}-2 \frac{D_{e} \alpha q e^{-\alpha r}}{1-e^{-\alpha r}}\right. \\
& \left.+\frac{D_{e} q[1-q] e^{-2 \alpha r}}{\left[1-e^{-\alpha r}\right]^{2}}-2 \frac{D_{e} \alpha q^{2} e^{-3 \alpha r}}{\left[1-e^{-\alpha r}\right]^{3}}\right) \frac{\mathbf{L} \Theta}{2 r}+O\left(\Theta^{2}\right)
\end{aligned}
$$

Now, we apply the Greene and Aldrich approximation for the centrifugal term in the perturbed potential $V_{n r-p e r t}^{\mathrm{hmp}}(s)$, we obtain:

$$
\begin{aligned}
V_{n r-p e r t}^{\mathrm{hmp}}(s) & =\left(\frac{l[l+1] \alpha^{4}}{[1-s]^{4}}-\frac{\alpha}{2}\left[\frac{a}{\{1-s\}^{3}}-\frac{b \alpha s}{\{1-s\}^{3}}+\left\{2 D_{e} q-b\right\} \frac{\alpha s}{\{1-s\}^{2}}\right.\right. \\
& \left.\left.+2 \frac{D_{e} \alpha q\{1-q\} s^{2}}{\{1-s\}^{3}}-2 \frac{D_{e} \alpha q^{2} s^{3}}{\{1-s\}^{4}}\right]\right) \mathbf{L} \Theta+O\left(\Theta^{2}\right) .
\end{aligned}
$$




\subsection{Nonrelativistic bound state correction under the improved Hellmann-generalized Morse potential}

In this subsection, we want to generate nonrelativistic bound state corrections under improved Hellmann-generalized Morse potential. The expression of perturbative potential in Eq. (89) needs to calculate the expectation values of $\left(1 /[1-s]^{4}\right)$, $\left(1 /[1-s]^{3}\right),\left(s /[1-s]^{3},\left(s /[1-s]^{2}\right),\left(s^{2} /[1-s]^{3}\right)\right.$ and $\left(s^{3} /[1-s]^{4}\right)$ to find the nonrelativistic energy corrections produced by the perturbative potential $V_{n r-p e r t}^{\mathrm{hmp}}(s)$. We have seen the expectation values of these terms in Eqs. (35). $\left.\mathrm{i}=1,2,3,4,5,6\right)$, allow us to get the global nonrelativistic expectation $\langle Z\rangle_{(n l m)}^{N R H M P}\left(n, n, \alpha, a, b, D_{e}, r_{e}\right)$ value is determined from the following expression:

$$
\begin{aligned}
\langle Z\rangle_{(n l m)}^{N R H M P}\left(n, \alpha, a, b, D_{e}, r_{e}\right) & =\left\langle l(l+1) \alpha^{4}\left\langle\frac{1}{(1-s)^{4}}\right\rangle_{(n l m)}-\frac{\alpha}{2}\left[a\left\langle\frac{1}{(1-s)^{3}}\right\rangle_{(n l m)}\right.\right. \\
& -b\left\langle\frac{s}{(1-s)^{3}}\right\rangle_{(n l m)}+\left(2 D_{e} q-b\right)\left\langle\frac{s^{3 / 2}}{(1-s)^{4}}\right\rangle_{(n l m)} \\
& \left.\left.+2 \alpha D_{e} q(1-q)\left\langle\frac{s^{2}}{(1-s)^{3}}\right\rangle_{(n l m)}-2 \alpha D_{e} q^{2}\left\langle\frac{s^{3}}{(1-s)^{4}}\right\rangle_{(n l m)}\right]\right) .
\end{aligned}
$$

And by following the same method used in the relativistic study, we obtain the nonrelativistic energy corrections $\Delta E_{h m p}^{t o t-n r}$ $\left(n, \alpha, a, b, D_{e}, r_{e}, \Theta, \sigma, \chi, j, l, s, m\right)$ for the generalized excited states due to the spin-orbit coupling, improved Zeeman effect and nonrelativistic perturbed Fermi gas potential under the influence of the improved Hellmann-generalized Morse potential in ENRQM symmetries are as follows:

$$
\Delta E_{h m p}^{t o t-n r}\left(n, \alpha, a, b, D_{e}, r_{e}, \Theta, \sigma, \chi, j, l, s, m\right)=\langle Z\rangle_{(n l m)}^{N R H M P}\left(n, \alpha, a, b, D_{e}, r_{e}\right)(\Theta \tau(j, l, s)+\sigma \aleph m+\chi \Omega m) .
$$

According to the standard perturbation theory. The new generalized nonrelativistic energy $E_{n r-n c}^{h m p}\left(n, n, \alpha, a, b, D_{e}, r_{e}, \Theta\right.$, $\sigma, \chi, j, l, s, m)$ for the excited $n^{t h}$ states, which, produced by the effect of the improved Hellmann-generalized Morse potential, is the sum of the nonrelativistic energies $E_{n l}^{n r}$ (see Eq. (16.2)) due to the effect of Hellmann-generalized Morse potential in NRQM and the above corrections in Eq. (91):

$$
E_{n r-n c}^{h m p}\left(n, \alpha, a, b, D_{e}, r_{e}, \Theta, \sigma, \chi, j, l, s, m\right)=E_{n l}^{n r}+\langle Z\rangle_{(n l m)}^{N R H M P}\left(n, \alpha, a, b, D_{e}, r_{e}\right)(\Theta \tau(j, l, s)+\sigma \aleph m+\chi \Omega m) .
$$

\subsection{Nonrelativistic particular cases under the IHGMP}

After examining the bound state solutions of any $l$-state deformed Schrödinger equation with the improved Hellmann-generalized Morse potential, our task is now to discuss some particular cases below. By adjusting the potential parameters for each case, some familiar potentials, which are useful for other physical systems, can be obtained.

1. Setting $D_{e}$ to zero the potential in Eq. (8) turns to the Hellmann potential (Eq. (48)) in NRQM symmetries. The perturbed effective potential in Eq. (89) turns to perturbed effective potential $V_{n r-p e r t}^{h p}(s)$ in the symmetries of RNCQM as follows:

$$
V_{n r-p e r t}^{h p}(s)=\frac{l(l+1) \alpha^{4}}{(1-s)^{4}}-\frac{\alpha}{2}\left(\frac{a}{(1-s)^{3}}-\frac{b \alpha s}{(1-s)^{3}}-b \frac{\alpha s}{(1-s)^{2}}\right) \mathbf{L} \Theta+O\left(\Theta^{2}\right) .
$$

The new relativistic energy in Eq. (92) reduces to the new energy $E_{n r-n c}^{h p}(n, \alpha, a, b, \Theta, \sigma, \chi, j, l, s, m)$ under improved equal scalar and vector improved Hellmann potential in ENRQM symmetries, as follows:

$$
E_{n r-n c}^{h p}(n, \alpha, a, b, \Theta, \sigma, \chi, j, l, s, m)=E_{h p-n l}^{n r}+\langle Z\rangle_{(n l m)}^{N R H P}(n, n, \alpha, a, b)(\Theta \tau(j, l, s)+\sigma \aleph m+\chi \Omega m),
$$

where $\langle Z\rangle_{(n l m)}^{N R H P}(n, n, \alpha, a, b)$ is determined as a particular case from the global nonrelativistic expectation value $\langle Z\rangle_{(n l m)}^{N R H P}$ $\left(n, n, \alpha, a, b, D_{e}, r_{e}\right)$ :

$$
\begin{aligned}
\langle Z\rangle_{(n l m)}^{N R H M P}(n, \alpha, a, b) & =\left\langle l(l+1) \alpha^{4}\left\langle\frac{1}{(1-s)^{4}}\right\rangle_{(n l m)}-\frac{\alpha}{2}\left[a\left\langle\frac{1}{(1-s)^{3}}\right\rangle_{(n l m)}\right.\right. \\
& \left.\left.-b\left\langle\frac{s}{(1-s)^{3}}\right\rangle_{(n l m)}-b\left\langle\frac{s^{3 / 2}}{(1-s)^{4}}\right\rangle_{(n l m)}\right]\right)
\end{aligned}
$$


while $E_{h p-n l}^{n r}$ can be obtained directly from Eq. (16.1) by replace $D_{e}$ with zero.

2. Setting $a=b=0$, the potential in Eq. (8) turns to the generalized Morse (Eq. (54)). The perturbed effective potential in Eq. (33) turns to perturbed effective potential $V_{n r-p e r t}^{m p}(s)$ in the symmetries of RNCQM as follows:

$$
V_{n r-p e r t}^{m p}(s)=\left(\frac{l(l+1) \alpha^{4}}{(1-s)^{4}}-D_{e} q \alpha^{2}\left[\frac{\alpha s}{(1-s)^{2}}+\frac{(1-q) s^{2}}{(1-s)^{3}}-\frac{q s^{3}}{(1-s)^{4}}\right]\right) \mathbf{L} \Theta+O\left(\Theta^{2}\right) .
$$

In this case, the nonrelativistic energy correction under the influence of improved Morse potentials in ENRQM symmetries is given by:

$$
\Delta E_{m p}^{t o t-n r}\left(n, \alpha, D_{e}, r_{e}, \Theta, \sigma, \chi, j, l, s, m\right)=\langle Z\rangle_{(n l m)}^{N R M P}\left(n, \alpha, D_{e}, r_{e}\right)(\Theta \tau(j, l, s)+\sigma \aleph m+\chi \Omega m) .
$$

Thus, the corresponding global expectation value $\langle Z\rangle_{(n l m)}^{N R M P}\left(n, n, \alpha, D_{e}, r_{e}\right)$ is determined from the following expression:

$$
\begin{aligned}
\langle Z\rangle_{(n l m)}^{N R M P}\left(n, \alpha, D_{e}, r_{e}\right) & =\left(l(l+1) \alpha^{4}\left\langle\frac{1}{(1-s)^{4}}\right\rangle_{(n l m)}-\alpha^{2} D_{e} q\left[b\left\langle\frac{s^{3 / 2}}{(1-s)^{4}}\right\rangle_{(n l m)}\right.\right. \\
& \left.\left.+(1-q)\left\langle\frac{s^{2}}{(1-s)^{3}}\right\rangle_{(n l m)}-q\left\langle\frac{s^{3}}{(1-s)^{4}}\right\rangle_{(n l m)}\right]\right) .
\end{aligned}
$$

The new nonrelativistic energy in Eq. (92) reduces to the new energy under IHGMP in ENRQM symmetries, as follows:

$$
E_{n r-n c}^{m p}\left(n, \alpha, D_{e}, r_{e}, \Theta, \sigma, \chi, j, l, s, m\right)=E_{m p-n l}^{n r}+\langle Z\rangle_{(n l m)}^{N R M P}\left(n, \alpha, D_{e}, r_{e}\right)(\Theta \tau(j, l, s)+\sigma \aleph m+\chi \Omega m),
$$

while $E_{m p-n l}^{n r}$ can be obtained directly from Eq. (16.1) by replace $a=b=0$ with zero.

\subsection{Study the nonrelativistic fermion cases}

We have seen that in the previous section that the eigenvalues of the operator $G^{2}=J^{2}-L^{2}-S^{2}$, for the case of spin-1/2, are determined by Eq. (80.1) thus, the nonrelativistic energy in Eq. (92) can be generalized to the case of spin-1/2 with an improved Hellmann-generalized Morse potential, in the symmetries of ENRQM, corresponding to the generalized excited states:

$$
\begin{aligned}
E_{n r-n c}^{h m p}\left(n, \alpha, a, b, D_{e}, r_{e}, \Theta, \sigma, \chi, j, l, s, m\right) & =D_{e}-\alpha a+\frac{\alpha^{2} l(l+1)}{2 M}-\frac{\alpha^{2}}{8 M}\left(\frac{\Lambda(n, l)-2 M \eta(l)}{\Lambda(n, l)}\right)^{2} \\
+\langle Z\rangle_{(n l m)}^{N R H M P}\left(n, \alpha, a, b, D_{e}, r_{e}\right) & \left\{\begin{array}{l}
\frac{l}{2}+\sigma \aleph m+\chi \Omega m \\
\text { For up polarity } j=l+1 / 2 \\
-\frac{l+1}{2}+\sigma \aleph m+\chi \Omega m \\
\text { For dawn polarity } j=l-1 / 2
\end{array}\right.
\end{aligned}
$$

We conclude with all merit that the combination of potentials in the new symmetries of ENRQM brought about an upgrade to the ordinary Schrödinger equation to become similar in the description ability of the Dirac equation. The spin condition was clearly shown in the above result in Eq. (98).

\subsection{Composite systems in ENRQM symmetries}

Now, considering composite systems such as molecules made of $N=2$ particles of masses $m_{n}(n=1,2)$ in the frame of noncommutative algebra, it is worth taking into account features of descriptions of the systems in the space. In NRQM symmetries, it was obtained that composite systems with different masses are described with different noncommutative parameters $[49,52,54,104]$ :

$$
\left[x_{n c \mu}(S, H, I) * x_{n c \nu}(S, H, I)\right]=i \theta_{\mu \nu}^{c} .
$$

with

$$
\theta_{\mu \nu}^{c}=\sum_{n=1}^{2} \mu_{n}^{2} \theta_{\mu \nu}^{(n)}
$$


with $\mu_{n}=\left(m_{n} / \sum_{n} m_{n}\right)$, the indices $(n=1,2)$ label the particle, and $\theta_{\mu \nu}^{(n)}$ is the parameter of noncommutativity, corresponding to the particle of mass $m_{n}$. Note that in the case of a system of two particles with the same mass $m_{1}=m_{2}$ such as the homogeneous $\left(\mathrm{N}_{2}, \mathrm{H}_{2}\right.$ and $\left.\mathrm{I}_{2}\right)$ diatomic molecules the parameter $\theta_{\mu \nu}^{(n)}=\theta_{\mu \nu}$. Thus, the two parameters $\Theta$ and $\sigma$ which appears in Eq. (92) are changed to the new form:

$$
\begin{aligned}
\Theta^{c 2} & =\left(\sum_{n=1}^{2} \mu_{n}^{2} \Theta_{12}^{(n)}\right)^{2}+\left(\sum_{n=1}^{2} \mu_{n}^{2} \Theta_{23}^{(n)}\right)^{2}+\left(\sum_{n=1}^{2} \mu_{n}^{2} \Theta_{13}^{(n)}\right)^{2}, \\
\sigma^{c 2} & =\left(\sum_{n=1}^{2} \mu_{n}^{2} \sigma_{12}^{(n)}\right)^{2}+\left(\sum_{n=1}^{2} \mu_{n}^{2} \sigma_{23}^{(n)}\right)^{2}+\left(\sum_{n=1}^{2} \mu_{n}^{2} \sigma_{13}^{(n)}\right)^{2} .
\end{aligned}
$$

and

$$
\chi^{c 2}=\left(\sum_{n=1}^{2} \mu \chi_{12}^{(n)}\right)^{2}+\left(\sum_{n=1}^{2} \mu \chi_{23}^{(n)}\right)^{2}+\left(\sum_{n=1}^{2} \mu \chi_{13}^{(n)}\right)^{2} .
$$

As it is mentioned above, in the case of a system of two particles with the same mass $m_{1}=m_{2}$ such as the homogeneous $\left(\mathrm{N}_{2}, \mathrm{H}_{2}\right.$ and $\left.\mathrm{I}_{2}\right)$ diatomic molecules $\Theta_{\mu \nu}^{(n)}=\Theta_{\mu \nu}$ and $\sigma_{\mu \nu}^{(n)}=\sigma_{\mu \nu}$. Finally, we can generalize the nonrelativistic global energy

$E_{n r-n c}^{h m p}\left(n, \alpha, A, B, V_{0}, \Theta, \sigma, \chi, j, l, s, m\right)$ under the improved Hellmann-generalized Morse potential model considering that composite systems with different masses are described with different noncommutative parameters for the diatomic $(\mathrm{HCl}, \mathrm{LiH}$, $\mathrm{ScH}, \mathrm{TiH}, \mathrm{VH}, \mathrm{CrH}, \mathrm{CuLi}, \mathrm{TiC}, \mathrm{NiC}, \mathrm{ScN}$ and $\mathrm{ScF}$ ) as:

$$
\begin{aligned}
E_{n r-n c}^{h m p}\left(n, \alpha, a, b, D_{e}, r_{e}, \Theta, \sigma, \chi, j, l, s, m\right) & =E_{n l}^{n r}+\langle Z\rangle_{(n l m)}^{N R H M P}\left(n, n, \alpha, a, b, D_{e}, r_{e}\right) \\
& \times\left(\Theta^{c} \tau(j, l, s)+\sigma^{c} \aleph m+\chi^{c} \Omega m\right) .
\end{aligned}
$$

Finally, we arrived at the important results achieved in this new work. The KGE, as the most well-known relativistic wave equation, describes spin-zero particles, but its extension to the RNCQM symmetric deformed Klein-Gordon equation under improved Hellmann-generalized Morse potential has a physical behavior similar to the Duffin-Kemmer equation for a meson with spin-s that can describe a dynamic state of a particle with spin one in the symmetries of relativistic noncommutative quantum mechanics. This is one of the most important new results of this research. It is worthwhile to mention that for the two simultaneous limits $(\Theta, \sigma, \chi)$ and $\left(\Theta^{c}, \sigma^{c}, \chi^{c}\right) \rightarrow(0,0,0)$ we recover the results of Refs. [34,35].

\section{Summary and Conclusions}

This main part of our paper gives a summary of the basic points in our work. In this work, we have found the approximate bound state solutions of the deformed Klein-Gordon, deformed Dirac and Schrödinger equations of the improved Hellmanngeneralized Morse potential, which correspond to high and low energy physics for the diatomic molecules $(\mathrm{HCl}, \mathrm{LiH}, \mathrm{H} 2$, $\mathrm{ScH}, \mathrm{TiH}, \mathrm{VH}, \mathrm{CrH}, \mathrm{CuLi}, \mathrm{TiC}, \mathrm{NiC}, \mathrm{ScN}$ and $\mathrm{ScF}$ ), in the symmetries of extended KG theory, Dirac theory and Schrödinger theory. We have used Bopp's shift method, stationary, perturbation theory, and the improved approximation scheme to deal with the centrifugal term. In addition to the usual state numbers $(n, l)$, the new energy equations have appeared sensitive to discrete atomic numbers $(j, l, s, m)$, the parameters for the quantum states $\left(\alpha, a, b, D_{e}, r_{e}\right)$ in addition to noncommutativity parameters $(\Theta, \sigma$ and $\chi)$. This new behavior is in the symmetries of extended relativistic and relativistic quantum mechanics equivalent to a conventional physical system under the influence of at least three perturbative systems with the perturbed spinorbit, improved Zeeman effect and the perturbed Fermi gas effect. The main difference lies in the fact that these perturbations appear automatically in the case of the new system of ERQM and ENRQM symmetries. Furthermore, we can conclude that the deformed Klein-Gordon equation under the improved Hellmann-generalized Morse potential becomes similar to DuffinKemmer equation for a meson with spin-s, it can describe the dynamic state of a particle with spin-s in this symmetry. We also noted how the MHGPs model can be reduced to the improved Hellmann potential and improved Morse potential by applying appropriate potential constant values. Moreover, we have applied our results to composite systems such as molecules made of $N=2$ particles of masses $m_{n}(n=1,2)$ such as $\mathrm{HCl}, \mathrm{LiH}, \mathrm{ScH}, \mathrm{TiH}, \mathrm{VH}, \mathrm{CrH}, \mathrm{CuLi}, \mathrm{TiC}, \mathrm{NiC}, \mathrm{ScN}$ and $\mathrm{ScF}$. It is worth mentioning that, for all cases, to make the two simultaneous limits $(\Theta, \sigma, \chi)$ and $\left(\Theta^{c}, \sigma^{c}, \chi^{c}\right) \rightarrow(0,0)$, the ordinary physical quantities are recovered in refs. [34,35]. Finally, given the effectiveness of the methods that we followed in achieving our goal in this research, we advise researchers to apply the same methods in other studies, whether in the relativistic and nonrelativistic regimes for others potentials. 


\section{Acknowledgments}

This work was supported by the Research Fund of Laboratory of Physics and Material Chemistry, University of
M'sila, and DGRSDT in Algeria with the project number B00L02UN280120180001. The author wishes to give their sincere gratitude to the referees for their kind comments which improved the manuscript.
1. E. Schrödinger, An Undulatory Theory of the Mechanics of Atoms and Molecules, Phys. Rev. 28 (1926) 1049, https: //doi.org/10.1103/PhysRev.28.1049

2. O. Klein, Quantentheorie und fünfdimensionale Relativitätstheorie, Z. Physik 37 (1926) 895, https: //doi.org/10.1007/BF01397481

3. W. Gordon, Der Comptoneffekt nach der Schrödingerschen Theorie, Z. Physik 40 (1926) 117, https : / / doi .org/10. $1007 / \mathrm{BF} 01390840$

4. N. Kemmer, The Particle Aspect of Meson Theory, Proc. R. Soc. Lond. A 173 (1939) 91, https://doi.org/10. $1098 /$ rspa.1939.0131.

5. P.A.M. Dirac, The Quantum Theory of the Electron, Proc. R. Soc. Lond. A 117 (1928) 610, https://doi.org/10. $1098 /$ rspa.1928.0023.

6. H. Hellmann, A combined approximation procedure for calculation of energies in the problem of many electrons, Acta Physicochim, U.R.S.S. 1 (1935) 913; ibid. 4 (1936) 225; 4 (1936) 324.

7. H. Hellmann, A New Approximation Method in the Problem of Many Electrons, J. Chem. Phys. 3 (1935) 61, https: //doi.org/10.1063/1.1749559

8. H. Hellmann and W. Kassatotchkin, Metallic Binding According to the Combined Approximation Procedure, J. Chem. Phys. 4 (1936) 324, https://doi.org/10.1063/1. 1749851

9. H. Yukawa, On the interaction of elementary particles I, Proc. Phys. Math. Soc. Japan 17 (1935) 48, https: //doi.org/ 10.11429/ppmsj1919.17.0_48

10. S.M. Ikhdair and R. Sever, A perturbative treatment for the bound states of the Hellmann potential, J. Mol. Struct. 809 (2007) 103, https: / doi.org/10.1016/j. theochem.2007.01.019

11. G. Kocak, O. Bayrad and I. Boztosum, Arbitrary 1-state solutions of the Hellmann potential, J. Theor. Comput. Chem. 06 (2007) 893, https://doi.org/10.1142/ S0219633607003313

12. S.M. Ikhdair, and B.J. Falaye. Two Approximate Analytic Eigensolutions of the Hellmann Potential with any Arbitrary Angular Momentum, Z. Naturforsch. A 68 (2013) 701, https://doi.org/10.5560/zna.2013-0054

13. J.C. Philips, and L. Kleinmann, New Method for Calculating Wave Functions in Crystals and Molecules, Phys. Rev. A 116 (1959) 287; https://doi.org/10.1103/PhysRev. 116.287, L. Kleinman and J. C. Phillips, Crystal Potential and Energy Bands of Semiconductors. III. Self-Consistent Calculations for Silicon, Phys. Rev. 118 (1960) 1153, https: //doi.org/10.1103/PhysRev.118.1153.
14. A.J. Hughes, and J. Callaway, Energy Bands in Body-Centered and Hexagonal Sodium, Phys. Rev. A 136 (1964) A1390, https://doi.org/10.1103/PhysRev.136.A1390

15. Y.P. Varshni, and R.C. Shukla, Alkali Hydride Molecules: Potential Energy Curves and the Nature of their Binding, Rev. Mod. Phys. 35 (1963) 130, https : / doi .org/10.1103/ RevModPhys.35.130

16. J.N. Das, and S. Chakraborty, Atomic inner-shell ionization, Phys. Rev. A 32 (1985) 176, https://doi.org/10. $1103 /$ PhysRevA. 32.176

17. M. Mousavi and M.R. Shojaei, Mirror Nuclei of $17 \mathrm{O}$ and $17 \mathrm{~F}$ in Relativistic and Nonrelativistic Shell Model, Adv. High Energy Phys. 2017 (2017) 5841701, https://doi.org/10. $1155 / 2017 / 5841701$

18. M. Hamzavi, K.E. Thylwe, and A.A. Rajabi, Approximate Bound States Solution of the Hellmann Potential. Commun. Theor. Phys. 60 (2013) 1, https://doi.org/10.1088/ 0253-6102/60/1/01.

19. C.A. Onate, M.C. Onyeaju, A.N. Ikot and O. Ebomwonyi, Eigen solutions and entropic system for Hellmann potential in the presence of the Shrodinger equation, Eur. Phys. J. Plus. 132 (2017) 462, https://doi.org/10.1140/ epjp/i2017-11729-8

20. C.O. Edet, K.O. Okorie, H. Louis and N.A. Nzeata-Ibe, Any 1-state solutions of the Schrödinger equation interacting with Hellmann-Kratzer potential model. Indian J. Phys. 94 (2020) 243, https://doi.org/10.1007/ s12648-019-01467-X

21. Z.H. Deng, and Y.P. Fan, A Potential Function of Diatomic Molecules, Shandong Univ. J. 7 (1957) 162.

22. P.M. Morse, Diatomic Molecules According to the Wave Mechanics. II. Vibrational Levels, Phys. Rev. 34 (1929) 57, https://doi.org/10.1103/PhysRev.34.57.

23. S.H. Dong, Factorization method in quantum mechanics Fundamental Theories in Physics. 150 (The Netherlands: Springer 2007) pp 187-213,

24. O.J. Oluwadare, K.J., Oyewumi, C.O. Akoshile and O.A. Babalola, Approximate analytical solutions of the relativistic equations with the Deng-Fan molecular potential including a Pekeris-type approximation to the (pseudo) centrifugal term, Physica Scripta, 86 (2012) 035002, https://doi.org/ 10.1088/0031-8949/86/03/035002

25. A.D.S. Mesa, C. Quesne and Y.F. Smirnov, Generalized Morse potential: Symmetry and satellite potentials, $J$. Phys. A 31 (1998) 321, https://doi.org/10.1088/ 0305-4470/31/1/028.

26. S.H. Dong and X.Y. Gu, Arbitrary 1 state solutions of the Schrödinger equation with the Deng-Fan molecular potential, J. Phys. Conf. Ser. 96 (2008) 012109, https://doi.org/ $10.1088 / 1742-6596 / 96 / 1 / 012109$ 
27. S.H. Dong, Relativistic Treatment of Spinless Particles Subject to a Rotating Deng-Fan Oscillator, Commun. Theor. Phys. 55 (2011) 969, https://doi.org/10.1088/ 0253-6102/55/6/05

28. H. Hassanabadi, B.H. Yazarloo, S. Zarrinkamar and H. Rahimov, Deng-Fan Potential for Relativistic Spinless Particlesan Ansatz Solution, Commun. Theor. Phys. 57 (2012) 339, https://doi.org/10.1088/0253-6102/57/3/02

29. S. Ortakaya, H. Hassanabadi and B.H. Yazarloo, Bound state solutions of the Dirac equation with the Deng-Fan potential including a Coulomb tensor interaction, Chin. Phys. B 23 (2014) 030306. https: / / doi .org/10 . 1088/ 1674-1056/23/3/030306

30. L.H. Zhang, X.P. Li and C.S. Jia, Approximate analytical solutions of the Dirac equation with the generalized Morse potential model in the presence of the spin symmetry and pseudospin symmetry, Phys. Scr. 80 (2009) 035003, https : / doi. org/10.1088/0031-8949/80/03/035003

31. E. Maghsoodi, H. Hassanabadi and S. Zarrinkamar, Spectrum of Dirac Equation Under Deng-Fan Scalar and Vector Potentials and a Coulomb Tensor Interaction by SUSYQM, FewBody Syst. 53 (2012) 525, https://doi.org/10.1007/ s00601-012-0314-5.

32. K.J. Oyewumi, O.J. Oluwadare, K. D. Sen and O.A. Babalola, Bound state solutions of the Deng-Fan molecular potential with the Pekeris-type approximation using the NikiforovUvarov (N-U) method, J. Math. Chem. 51 (2012) 976, https : //doi.org/10.1007/s10910-012-0123-6.

33. E. Omugbe, Non-relativistic Energy Spectrum of the DengFan Oscillator via the WKB Approximation Method, Asian J. Phys. Chem. Sci. 8 (2020) 26, https : / doi.org/10. 9734/ajopacs/2020/v8i130107

34. P.O. Okoia, C.O. Edetb and T.O. Magu, Relativistic treatment of the Hellmann-generalized Morse potential, Rev. Mex. Fis. 66 (2020) 1, https://doi.org/10.31349/RevMexFis. 66.1

35. O. Ebomwonyi, C.A. Onate, M.C. Onyeaju and A.N. Ikot, Any $l$ - states solutions of the Schrödinger equation interacting with Hellmann-generalized Morse potential model, Karbala Int. J. Mod. Sci. 3 (2017) 59, https://doi.org/10.1016/j. kijoms.2017.03.001

36. H.S. Snyder, The Electromagnetic Field in Quantized SpaceTime, Phys. Rev. 72 (1947) 68, https://doi.org/10. 1103/PhysRev. 72.68

37. A. Connes, M. R. Douglas and A. Schwarz, Noncommutative geometry and Matrix theory, J. High Energy Phys. 1998 (1998) 003. https://doi.org/10.1088/ 1126-6708/1998/02/003.

38. N. Seiberg and E. Witten, String theory and noncommutative geometry, J. High Energy Physics 1999 (1999) 032, https : //doi.org/10.1088/1126-6708/1999/09/032

39. S. Capozziello, G. Lambiase and G. Scarpetta, Generalized uncertainty principle from quantum geometry. Int. J. Theor. Phys. 39 (2000) 15, https://doi.org/10.1023/A: 1003634814685

40. S. Doplicher, K. Fredenhagen and J.E. Roberts, Spacetime quantization induced by classical gravity, Phys. Lett.
B 331 (1994) 39, https://doi.org/10.1016/ 0370-2693(94) 90940-7.

41. E. Witten, Refection on the fate spacetime, Phys. Today 49 (1996) 24,https://doi.org/10.1063/1.881493

42. A. Kempf, G. Mangano and R.B. Mann, Hilbert space representation of the minimal length uncertainty relation, Phys. Rev. D 52 (1995) 1108, https://doi.org/10.1103/ PhysRevD.52.1108

43. F. Scardigli, Some heuristic semi-classical derivations of the Planck length, the Hawking effect and the unruh effect, Nuovo Cimento B 110 (1995) 1029, https://doi.org/ $10.1007 / \mathrm{BF} 02726152$.

44. R.J. Adler and D.I. Santigo, On gravity and the uncertainty principal, Mod. Phys. Lett. A 14 (1999) 1371, https : / / doi . org/10.1142/S0217732399001462

45. T. Kanazawa, G. Lambiase, G. Vilasi and A. Yoshioka, Noncommutative Schwarzschild geometry and generalized uncertainty principle, Eur. Phys. J. C. 79 (2019) 95, https:// doi.org/10.1140/epjc/s10052-019-6610-1.

46. F. Scardigli, Generalized uncertainty principle in quantum gravity from micro-black hole Gedanken experiment, Phys. Lett. B 452 (1999) 39, https://doi.org/10.1016/ S0370-2693(99)00167-7

47. P.M. Ho and H.C. Kao, Noncommutative quantum mechanics from noncommutative quantum field Theory, Phys. Rev. Lett. 88 (2002) 151602. https://doi.org/10.1103/ PhysRevLett.88.151602.

48. O. Bertolami, G.J. Rosa, C.M.L. Dearagao, P. Castorina and D. Zappala, Scaling of variables and the relation between noncommutative parameters in noncommutative quantum mechanics, Mod. Phys. Lett. A 21 (2006) 795, https: / / doi.org/10. $1142 / \mathrm{S} 0217732306019840$

49. P. Gnatenko, Parameters of noncommutativity in Liealgebraic noncommutative space, Phys. Rev. D 99 (2019) 026009, https://doi.org/10.1103/PhysRevD.99. 026009 .

50. O. Bertolami and P. Leal, Aspects of phase-space noncommutative quantum mechanics, Phys. Lett. B 750 (2015) 6, https : //doi.org/10.1016/j.physletb.2015.08.024.

51. M.A. De Andrade and C. Neves, Noncommutative mapping from the symplectic formalism, J. Math. Phys. 59 (2018) 012105, https://doi.org/10.1063/1.4986964

52. Kh.P. Gnatenko and V.M. Tkachuk, Upper bound on the momentum scale in noncommutative phase space of canonical type, (Europhys. Lett.) 127 (2019) 20008, https://doi. org/10.1209/0295-5075/127/20008

53. Kh.P. Gnatenko and TV.M. kachuk, Composite system in rotationally invariant noncommutative phase space, Int. J. Mod. Phys. A 33 (2018) 1850037, https : / / doi . org/10. $1142 / \mathrm{S} 0217751 \times 18500379$

54. A. Maireche, Bound-state solutions of the modified KleinGordon and Schrödinger equations for arbitrary 1-state with the modified Morse potential in the symmetries of noncommutative quantum mechanics, J. Phys. Stud. 25 (2021) 1002, https://doi.org/10.30970/jps.25.1002 
55. A. Maireche, A Theoretical Model of Deformed Klein-Gordon Equation with Generalized Modified Screened Coulomb Plus Inversely Quadratic Yukawa Potential in RNCQM Symmetries, Few-Body Syst 62 (2021) 12.https : / doi .org/10. 1007/s00601-021-01596-2

56. A. Maireche, Solutions of Klein-Gordon equation for the modified central complex potential in the symmetries of noncommutative quantum mechanics, Sri Lankan J. Phys. 22 (2021) 1, Doi http://doi.org/10.4038/sljp.v22i1.8079

57. A. Maireche, Theoretical Investigation of the Modified Screened cosine Kratzer potential via Relativistic and Nonrelativistic treatment in the NCQM symmetries, Lat. Am. J. Phys. Educ. 14 (2020) 3310.

58. A. Maireche, Modified unequal mixture scalar vector HulthénYukawa potentials model as a quark-antiquark interaction and neutral atoms via relativistic treatment using the improved approximation of the centrifugal term and Bopp's shift method, Few-Body Syst. 61 (2020) 30. https://doi.org/10. 1007/s00601-020-01559-z.

59. J. Gamboa, M. Loewe and J.C. Rojas, Noncommutative quantum mechanics, Phys. Rev. D. 64 (2001) 067901. https: //doi.org/10.1103/PhysRevD.64.067901

60. A. Maireche, A New Approach to the approximate analytic solution of the three-dimensional Schrödinger equation for Hydrogenic and neutral atoms in the generalized Hellmann potential model Ukr. J. Phys. 65 (2020) 987. https : / / doi . org/ 10.15407/ujpe65.11.987

61. A. Maireche, A New Look at a Nonrelativistic Shell Model: Study of the Mirror Nuclei $17 \mathrm{O}$ and $17 \mathrm{~F}$ in the Symmetries of NCQM, To Phys. J. 5 (2020) 51.

62. A. Maireche Any l-States solutions of the modified Schrodinger equation with generalized Hellmann-Kratzer potential model in the symmetries of NRNCQM, To Phys. J. 4 (2019) 16, https://purkh.com/index.php/tophy/ article/view/521.

63. E.F. Djemaï and H. Smail, On quantum mechanics on noncommutative quantum phase space, Commun. Theor. Phys. 41 (2004) 837, https://doi.org/10.1088/ 0253-6102/41/6/837

64. Y. Yi, K. Kang, W. Jian-Hua and C. Chi-Yi, Spin-1/2 relativistic particle in a magnetic field in NC phase space, Chin. Phys. C. 34 (2010) 543, https://doi.org/10.1088/ $1674-1137 / 34 / 5 / 005$

65. O.G. Valencia and H.L.A. Arias, Thermodynamic properties of diatomic molecule systems under SO $(2,1)$-anharmonic Eckart potential, Int. J. Quantum Chem. 118 (2018) e25589. https : //doi.org/10.1002/qua.25589

66. O. Bertolami, J.G. Rosa, C.M.L. de Aragão, P. Castorina and D. Zappalà, Noncommutative gravitational quantum well, Phys. Rev. D 72 (2005) 025010, https://doi.org/10.1103/ PhysRevD.72.025010

67. J. Zhang, Fractional angular momentum in non-commutative spaces, Phys. Lett. B 584 (2004) 204, https://doi.org/ $10.1016 / j \cdot$ physletb.2004.01.049

68. M. Chaichian, Sheikh-Jabbari and A. Tureanu, Hydrogen atom spectrum and the Lamb Shift in noncommutative QED, Phys. Rev. Lett. 86 (2001) 2716, https://doi.org/10.1103/ PhysRevLett.86.2716
69. E.M.C. Abreu, C. Neves and W. Oliveira, Noncommutativity from the symplectic point of view, Int. J. Mod. Phys. A 21 (2006) 5359, https://doi.org/10.1142/ S0217751X06034094

70. E.M.C. Abreu, J.A. Neto, A.C.R. Mendes C. Neves,W. Oliveira and M.V. Marcial, Lagrangian formulation for noncommutative nonlinear systems, Int. J. Mod. Phys. A 27 (2012) 1250053, https://doi.org/10.1142/S0217751X12500534

71. J. Wang and K. Li, The HMW effect in noncommutative quantum mechanics, J. Phys. A Math. Theor. 40 (2007) 2197, https://doi.org/10.1088/1751-8113/40/ $9 / 021$

72. K. Li, and J. Wang, The topological AC effect on non-commutative phase space, Eur. Phys. J. C. $\mathbf{5 0}$ (2007) 1007, https://doi.org/10.1140/epjc/ s10052-007-0256-0

73. A. Maireche, A theoretical investigation of nonrelativistic bound state solution at finite temperature using the sum of modified Cornell plus inverse quadratic potential, Sri Lankan J. Phys. 21 (2020) 11, https://doi.org/10.4038/ sljp.v21i1.8069

74. A. Maireche, Extended of the Schrödinger Equation with New Coulomb Potentials plus Linear and Harmonic Radial Terms in the Symmetries of Noncommutative Quantum Mechanics, J. Nano- Electron. Phys. 10 (2018) 06015, https://doi. org/10.21272/jnep.10(6).06015

75. A. Maireche, Heavy light mesons in the symmetries of extended nonrelativistic quark model, Yanbu J. Eng. Sci. 17 (2019) 51, https://doi.org/10.53370/001c. 23732

76. A. Maireche, The Klein-Gordon equation with modified Coulomb plus inverse-square potential in the noncommutative three-dimensional space, Mod. Phys. Lett. A. 35 (2020) 052050015. https://doi.org/10.1142/ S0217732320500157

77. A. Maireche, The Klein-Gordon equation with modified Coulomb potential plus inverse-square-root potential in three-dimensional noncommutative space, To Phys. J. 3 (2019) 186, https://purkh.com/index.php/ tophy/article/view/489

78. A. Maireche, Bound state solutions of Klein-Gordon and Schrödinger equations with linear combination of Hulthén and Kratzer potentials, Afr. Rev. Phys. 15 (2020) 19, http://lamp.ictp.it/index.php/aphysrev/ article/view/1779/620.

79. H. Motavalli and A.R. Akbarieh, Klein-Gordon equation for the Coulomb potential in noncommutative space, Mod. Phys. Lett. A 25 (2010) 2523, https://doi.org/10.1142/ S0217732310033529

80. M. Darroodi, H. Mehraban and H. Hassanabadi, The KleinGordon equation with the Kratzer potential in the noncommutative space, Mod. Phys. Lett. A 33 (2018) 1850203, https: //doi.org/10.1142/S0217732318502036

81. A. Saidi and M.B. Sedra, Spin-one $(1+3)$-dimensional DKP equation with modified Kratzer potential in the noncommutative space, Mod. Phys. Lett. A 35 (2020) 2050014, https://doi.org/10.1142/S0217732320500145 
82. H. Aounallah and A. Boumali,, Solutions of the DuffinKemmer Equation in Non-Commutative Space of Cosmic String and Magnetic Monopole with Allowance for the Aharonov-Bohm and Coulomb Potentials, Phys. Part. Nucl. Lett. 16 (2019) 195, https://doi.org/10.1134/ S1547477119030038

83. A. Maireche, A model of modified Klein-Gordon equation with modified scalar-vector Yukawa potential, Afr. Rev Phys. 15 (2020) 1, http://lamp.ictp.it/index. php/aphysrev/article/view/1777/618

84. A. Maireche, The Relativistic and Nonrelativistic Solutions for the Modified Unequal Mixture of Scalar and Time-Like Vector Cornell Potentials in the Symmetries of Noncommutative Quantum Mechanics, Jordan J. Phys. 14 (2021) 59, https: //doi.org/10.47011/14.1.6

85. L. Mezincescu, Star Operation in Quantum Mechanics, https://arxiv.org/abs/hep-th/0007046.

86. L. Gouba, A comparative review of four formulations of noncommutative quantum mechanics, Int. J. Mod. Phys. A 31 (2016) 1630025, https://doi.org/10.1142/ S0217751X16300258

87. F. Bopp, La mécanique quantique est-elle une mécanique statistique classique particulière?, Ann. Inst. Henri Poincaré 15, (1956) 81.

88. A. Maireche, New Relativistic Bound States for Modified Pseudoharmonic Potential of Dirac Equation with Spin and Pseudo-Spin Symmetry in One-electron Atoms, Afr. Rev Phys. 12 (2017) 130, http://lamp.ictp.it/index.php/ aphysrev/article/view/1533/564

89. A. Maireche, New Relativistic Atomic Mass Spectra of Quark ( $\mathrm{u}, \mathrm{d}$ and $\mathrm{s}$ ) for Extended Modified Cornell Potential in Nano and Plank's Scales, J. Nano-Electron. Phys. 8 (2016) 01020, https://doi.org/10.21272/jnep.8(1).01020

90. A. Maireche, A New Relativistic Study for Interactions in One-electron atoms (Spin $\frac{1}{2}$ Particles) with Modified Mie-type Potential J. Nano- Electron. Phys. 8 (2016) 04027. https: //doi.org/10.21272/jnep.8(4(1)).04027.

91. A. Maireche, Investigations on the Relativistic Interactions in One-Electron Atoms with Modified Yukawa Potential for Spin 1/2 Particles Int. Front. Sci. Lett. 11 (201) 29, Doi https://doi.org/10.18052/www.scipress. Com/IFSL.11.29

92. A. Maireche, Nonrelativistic treatment of Hydrogen-like and neutral atoms subjected to the generalized perturbed Yukawa potential with centrifugal barrier in the symmetries of noncommutative Quantum mechanics, Int. J. Geom. Methods Mod. Phys. 17 (2020) 2050067, https://doi.org/10.1142/ S021988782050067X

93. A. Maireche, A Recent Study of Excited Energy Levels Diatomics for Modified more General Exponential Screened
Coulomb Potential: Extended Quantum Mechanics, J. NanoElectron. Phys. 9 (2017) 03021, https://doi.org/10. $21272 /$ jnep.9(3).03021.

94. A. Maireche, A new study of energy levels of hydrogenic atoms and some molecules for new more general exponential screened Coulomb potential, Open Acc J Math Theor Phy. 1 (2018) 232, Doi $10.15406 /$ oajmtp.2018.01.00040

95. A. Maireche, Effects of two-dimensional noncommutative theories on bound states Schrödinger diatomic molecules under New modified Kratzer-type interactions, Int. Lett. Chem. Phys. Astron. 76 (2017) 1, https://doi.org/10.18052/ WWW.scipress.com/ILCPA.76.1

96. R.L. Greene and C. Aldrich, Variational wave functions for a screened Coulomb potential, Phys. Rev. A 14 (1976) 2363, https://doi.org/10.1103/PhysRevA.14.2363.

97. M. Badawi, N. Bessis and G. Bessis, On the introduction of the rotation-vibration coupling in diatomic molecules and the factorization method, J. Phys. B 5 (1972) L157, https : / / doi . org/10.1088/0022-3700/5/8/004

98. W.C. Qiang, and S.H. Dong, Analytical approximations to the solutions of the Manning-Rosen potential with centrifugal term, Phys. Lett. A 368 (2007) 13, https : / / doi .org/10. 1016/j.physleta.2007.03.057

99. F.A. Serrano, X.Y. Gu and S.H. Dong, Qiang-Dong proper quantization rule and its applications to exactly solvable quantum systems, J. Math. Phys. 51 (2010) 082103, https: // doi.org/10.1063/1.3466802

100. S.-H. Dong, W.-C. Qiang, G.-H. Sun and V.B. Bezerra, Analytical approximations to the l-wave solutions of the Schrödinger equation with the Eckart potential, J. Phys. A: Math. Theor. 40 (2007) 10535, https://doi.org/10. 1088/1751-8113/40/34/010

101. Y. Zhang, Approximate analytical solutions of the KleinGordon equation with scalar and vector Eckart potentials, Phys. Scr. 78 (2008) 015006, https://doi.org/10.1088/ 0031-8949/78/01/015006

102. S. Medjedel and K. Bencheikh, Exact analytical results for density profile in Fourier space and elastic scattering function of a rotating harmonically confined ultra-cold Fermi gas, Phys. Lett. A 383 (2019) 1915, https://doi.org/10.1016/ j.physleta.2019.03.021

103. K. Bencheikh, S. Medjedel and G. Vignale, Current reversals in rapidly rotating ultracold Fermi gases, Phys. Rev. A 89 (2014) 063620, https://doi.org/10.1103/PhysRevA.89. 063620 .

104. K.P. Gnatenko, Composite system in noncommutative space and the equivalence principle, Phys. Lett. A 377 (2013) 3061, https://doi.org/10.1016/j.physleta.2013. 09.036 . 